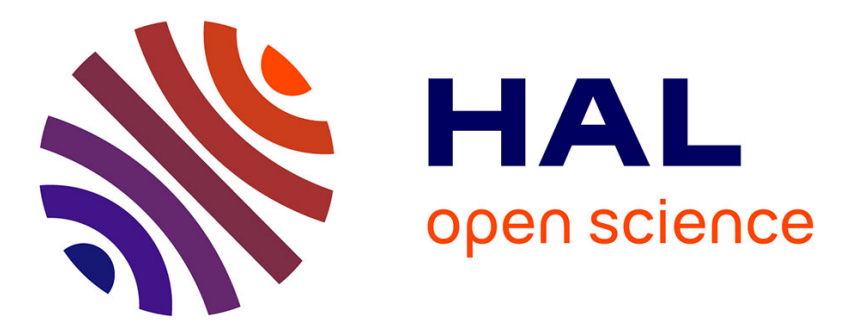

\title{
Global stability analysis of 3D micro-combustion model
}

Michele A. Bucci, Jean-Christophe Robinet, Sergio Chibbaro

\section{To cite this version:}

Michele A. Bucci, Jean-Christophe Robinet, Sergio Chibbaro. Global stability analysis of 3D micro-combustion model. Combustion and Flame, 2016, 167, pp.132-148.

10.1016/j.combustflame.2016.02.018 . hal-02454411

\section{HAL Id: hal-02454411 \\ https://hal.science/hal-02454411}

Submitted on 24 Jan 2020

HAL is a multi-disciplinary open access archive for the deposit and dissemination of scientific research documents, whether they are published or not. The documents may come from teaching and research institutions in France or abroad, or from public or private research centers.
L'archive ouverte pluridisciplinaire HAL, est destinée au dépôt et à la diffusion de documents scientifiques de niveau recherche, publiés ou non, émanant des établissements d'enseignement et de recherche français ou étrangers, des laboratoires publics ou privés. 


\title{
Global stability analysis of 3D micro-combustion model
}

\author{
Michele A. Bucci ${ }^{\mathrm{a}, \mathrm{b}, \mathrm{c}, *}$, Jean-Christophe Robinet ${ }^{\mathrm{a}}$, Sergio Chibbaro ${ }^{\mathrm{b}}$ \\ a DynFluid Laboratory, EA92, Arts et Métiers ParisTech, 151, Bd. de l'Hopital, Paris 75013, France \\ b Sorbonne Universités, UPMC Univ Paris 06, CNRS, UMR 7190, Institut Jean Le Rond d'Alembert, Paris F-75005, France \\ c Dipartimento di Meccanica, Matematica e Management, Politecnico di Bari, via Re David 200, Bari 70125, Italy
}

Keywords:

Micro-combustion

Global stability

Flame dynamics

\begin{abstract}
A B S T R A C T
We report on the linear stability of micro-combustion in pipe, where two instabilities manifest at high and low flow-rates. The combustion of a stoichiometric methane/air premixed mixture has been numerically investigated within a 3D reduced model. This model reproduces decently the flame dynamics in the range of speed between $5-100 \mathrm{~cm} / \mathrm{s}$. The flame position, the stability thresholds of the Flame with Repetitive Extinction and Ignition (FREI) and the flame shape are in accordance with the experiments. Furthermore, an analysis of the integral values of all mechanisms involved in the flame evolution has been carried out near the two stability thresholds. The phase shift between the reaction term and the radial diffusion has been identified as the source of instability in both cases. The global behavior has been then investigated with a linear stability analysis. The 2D and 3D temperature and concentration perturbations have been found by solving the eigenvalue problem obtained by linearizing the model around the basic state. Only one unstable axisymmetric mode has been found. This is in agreement with the direct numerical simulation of the model.
\end{abstract}

\section{Introduction}

Improvement in fabrication techniques has rapidly affected the engineering standards in the last decades. Notably, starting from electronics, huge efforts toward the miniaturization of mechanical and electro-mechanical devices have been made. This trend also has recently included combustion [1,2]. In particular, remarkable efforts have been devoted to the design of microcombustors, which can enable the development of micro-power generation devices of low weight and long life [3]. The potential of such devices resides in the high energy density of hydrocarbon fuels, which would make a miniaturized device strongly competitive with the Lithium batteries currently available in the market $[4,5]$.

Variousattempts have been already made to design small-scale engines [3]. For instance, heat-recirculating combustors, such as the double-spiral counter-current Swiss roll [6,7], have been developed to sustain combustion under conditions that would lead to extinction without recirculation. Furthermore, several liquid hydrocarbons that supply rotary engines have been also tried [8].

Nonetheless, the practical performance of a microcombustor is especially constrained by both the low overall efficiency and the

\footnotetext{
* Corresponding author at: DynFluid Laboratory, EA92, Arts et Métiers ParisTech, 151, Bd. de l'Hopital, 75013 Paris, France.

E-mail address: Michele.BUCCI@ensam.eu (M.A. Bucci).
}

narrow range of operational conditions. In particular, the premixed flame propagation in such devices may exhibit different combustion modes, which may render any microcombustor design hard. In fact, the decrease of the ratio of the reacting volume to the wall surface can lead to higher heat losses, causing instabilities and extended flame thermal quenching. These new phenomena pose challenging issues to be understood to improve the combustor design.

Numerous numerical and experimental studies have analyzed the dynamics of reactive flames in narrow channels $[9,10]$ and circular ducts [11-14]. It is worth noting that experiments have been performed in pipes. A rich physics is observed with different regimes at varying diameter and mass flow-rate. Notably, under steady conditions, some studies reported steady mild or flameless combustion [15], while unsteady behaviors are also exhibited, such as periodic frequent repetitive ignition/extinction (FREI) [15-18], oscillating flames $[10,15,19]$ and spinning flames $[12,20,21]$. As pointed out in these studies, at some critical configurations with regard to the diameter and the mass flow-rate, complex instabilities grow up. This intriguing feature has motivated the analysis of such instabilities, and, in particular, reduced models of engineering interest have been used to explore their nature. In fact, there is not a consensus on the nature of these instabilities, some studies proposing a chemical mechanism [13], whereas other underline the main role of hydrodynamics [14]. It is therefore relevant to get insights from a fundamental study of such instabilities. 
Moreover, while reduced models have been shown to correctly capture the main physics of the problem with detailed chemistry [10] and even with one-step chemistry [14], the precise instability dynamics remains to be unfold. In this sense, first numerical studies have been carried out in a configuration idealizing experiments [22], a planar channel with a step-wise temperature and reduced chemistry. The study includes the linear-stability analysis of the 1D case [23]. More recently, a more complete 2D stability analysis has been conducted by Kurdyumov [24] and SánchezSanz et al. [25]. Their work investigates in detail the same model with a step-wise temperature, providing a stability map based upon the numerical simulation of the linearized equations; while the global stability analysis has been carried out, the eigenspectrum has not been extracted and only the most unstable mode has been searched. Moreover the stability analysis has been performed so far for the plane channel case. Conversely, in the experimental pipe cases, a natural periodicity in the azimuthal direction is present, and that surely has influenced the solution symmetries.

In the present work, we complete these studies, reporting on the global linear stability analysis of the 2D and 3D reduced models in a pipe flow. It is worth noting that our goal is not to propose a new model. The major result of the work is to provide the whole spectrum of modes, which allows the most accurate analysis of the instabilities. More in details, we compute the basic state of the flow through a selective frequency damping (SFD) method and, then, we compute numerically the whole spectrum of eigenvalues. The results are obtained both for the FREI instability and for the transition from the FREI regime to the stable weak one. All results are compared with those obtained via the numerical simulations of the full equations.

The paper is organized as follows: in Section 2, the 3D reduced model is presented, and it is shown how it can represent the different behaviors observed in the experiments. Linear stability analysis is then introduced. In this framework the basic state and the linearized model are obtained and shown. In Section 3, the numerical tools used to solve the model and the eigenvalue problem are described. Then, the unstable dynamics of the flame near the two stability thresholds is studied by performing the direct numerical simulation of the model. Moreover, a first characterization of the linear stability in term of growth rate and pulsation is accomplished always through the direct numerical simulation. These first results are then confirmed in Section 4 where the entire eigenspectrum of the most unstable modes for an axisymmetric perturbation is shown. The fluctuation fields of the temperature and concentration of $\mathrm{CH}_{4}$ are displayed and temporally reconstructed. These are the most important and novel results of the present work. In Section 4 the 3D non-axisymmetric mode are investigated. Finally, the most important results of this work are summarized and new outlooks are highlighted.

\section{Theoretical framework}

\subsection{Test-case and combustion model}

We consider a premixed stoichiometric mixture of air and methane flowing in a pipe with diameter $d=2 \mathrm{~mm}$, as shown in Fig. 1. At the wall, a temperature gradient $T_{w}(z)$ is imposed (Fig. 1) as done in recent experiments [15,18]. Notably, the temperature profile was reconstructed by acquiring the experimental points provided by Tsuboi et al. [18] and accurately interpolated with a polynomial of eleventh order. The temperature increases from $T_{\min }=300 \mathrm{~K}$ to $T_{\max }=1348 \mathrm{~K}$.

In this work, we focus on the reduced model discussed in [14], which we refer to for more details. The dynamics is described by only two scalar advection-diffusion-reaction equations, one for the combustible concentration and one for the temperature, the den-
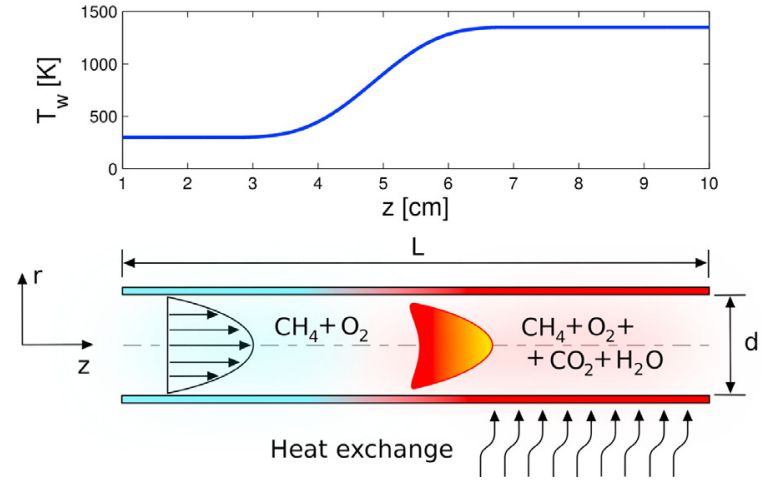

Fig. 1. Sketch of the geometry and temperature profile imposed at the wall.

sity of the gas $\rho$ is kept constant:

$\frac{\partial Y}{\partial t}+u_{z} \frac{\partial Y}{\partial z}=D\left[\frac{1}{r} \frac{\partial}{\partial r}\left(r \frac{\partial Y}{\partial r}\right)+\frac{1}{r^{2}} \frac{\partial^{2} Y}{\partial \theta^{2}}+\frac{\partial^{2} Y}{\partial z^{2}}\right]+S_{Y}$,
$\frac{\partial T}{\partial t}+u_{z} \frac{\partial T}{\partial z}=D\left[\frac{1}{r} \frac{\partial}{\partial r}\left(r \frac{\partial T}{\partial r}\right)+\frac{1}{r^{2}} \frac{\partial^{2} T}{\partial \theta^{2}}+\frac{\partial^{2} T}{\partial z^{2}}\right]+S_{T}$,

The imposed velocity is

$u_{r}=0$

$u_{\theta}=0$

$u_{z}=-\frac{1}{4 \eta} \frac{\partial p}{\partial z}\left(R^{2}-r^{2}\right)=-\frac{2 U_{0}}{R^{2}}\left(R^{2}-r^{2}\right)$

where $U_{0}$ is the mean axial speed, $R$ is the radius of the pipe, and $D$ is the diffusion coefficient taken to be constant. The production terms are as follows:

$S_{Y}=\frac{v_{\mathrm{CH}_{4}} W_{\mathrm{CH}_{4}}}{\rho} K_{f}=-A^{*} Y \exp \left(-\frac{T_{a}}{T}\right)$

$S_{T}=-\frac{Q}{C_{p}} S_{Y}$

where $Q$ is the heat of combustion, $C_{p}$ is the specific heat at constant pressure, and $v_{\mathrm{CH}_{4}}$ is the molecular stoichiometric coefficient and is taken equal to -1 . The ratio $Q / C_{p}=35,000 \mathrm{~K}$ is required to match the flame adiabatic temperature at constant pressure $\left(p_{0}=\right.$ $1 \mathrm{bar}, T_{0}=300 \mathrm{~K}$ ). A Lewis number equal to one is assumed. In the system (1a)-(1b) only the parameters $D$ and $A^{*}$ are undetermined and they have to be calibrated. Then, Bianco et al. [14] have shown that the model is able to correctly reproduce the phase-diagram of the flame-position versus the flow rate given by the experiments [18], assuming $D=0.6667 \mathrm{~cm}^{2} / \mathrm{s}$ and $A^{*}=1.455 \times 10^{9} \mathrm{~s}^{-1}$. Notably, the calibration was carried out by Bianco et al. [14] precisely to minimize the error on the flame position and on the upper and lower instability thresholds of the FREI regime.

The boundary conditions imposed on the temperature and concentration are:

- Inflow condition at $z=0$ :

$T(r, 0)=T^{0}=300 \mathrm{~K}, \quad Y_{\mathrm{CH}_{4}}(r, 0)=Y_{\mathrm{CH}_{4}}^{0}=0.055$

- Outflow condition at $z=L$ :

$\frac{\partial T(r, L)}{\partial z}=0, \quad \frac{\partial Y_{\mathrm{CH}_{4}}(r, L)}{\partial z}=0$

- Symmetric condition is applied at channel axis $r=0$ :

$\frac{\partial T(0, z)}{\partial r}=0, \quad \frac{\partial Y_{\mathrm{CH}_{4}}(0, z)}{\partial r}=0$ 
- At channel wall $r=R$

$$
T(R, z)=T_{w}(z), \quad \frac{\partial Y_{\mathrm{CH}_{4}}(R, z)}{\partial r}=0
$$

As pointed out by Tsuboi et al. [18], it is possible to neglect the influence of the flame temperature on the wall. However, possible difference between the diffused temperature near to the wall and $T_{w}$ represents the heat dissipation. Since radial terms are taken into account, the 2D model includes the exact heat balance, whereas it is necessary to add a supplementary term to represent the heat exchange with walls, in 1D models [13-15,18].

\subsection{Numerics}

The main purpose of this work is to carry out a complete linear stability analysis of the 2D model, Eqs. (1a) and (1b), which have therefore to be linearized around the basic state, that is the solution of the steady problem, as explained later. However, the linear results will be compared whenever possible with the numerical solution of the complete set of Eqs. (1a) and (1b). In this section, we briefly present the main numerical tools used.

The height of the domain is fixed by the experiments $[15,18]$ $R=1 \mathrm{~mm}$, and the length of the domain is taken equal to $L=$ $10 \mathrm{~cm}$. The temperature gradient at the wall is placed at the center of the domain, see Fig. 1 . The numerical domain is discretized with a Cartesian grid of $N_{z} \times N_{r}$ grid points along the cylinder length and radius, respectively, where $z(i) \in[0, L] \quad i=1, \ldots, N_{z}$ and $r(j) \in[0, R] \quad j=1, \ldots, N_{r}$ are the cylindrical coordinates of the generic grid point $(i, j)$. The mesh must be capable of capturing the smallest scales without filtering out the solution. Keeping the mesh constant over the entire domain, the solution is not dependent on the grid with a discretization size less than $0.02 \mathrm{~mm}$ in the $z$ direction. A minimum mesh size of $\Delta z=1.25 \times 10^{-2} \mathrm{~mm}$ was chosen. Along the $r$ direction, the gradients are less strong, then a coarse grid was used $\Delta r=2.5 \times 10^{-2} \mathrm{~mm}$. Following Bianco et al. [14], to avoid the large number of discretization points a moving mesh has been adopted. With this method only the region with strong gradients is finely discretized. Away from the flame, the mesh size becomes larger according to a geometric series. All results were obtained with a residual level less than $10^{-10}$. The quality of the stability analysis depends strongly on the quality of the spatial resolution of the basic state [26]. To avoid numerical errors, a sixthorder finite difference scheme was used for the spatial gradients. The numerical integration in time has been done with an explicit Runge-Kutta fourth-order scheme. Nevertheless, to compute the basic state a standard Euler upwind scheme is used.

\subsection{Phenomenology}

To better understand the stability analysis, we summarize here the basic physical behavior of the system, as obtained solving numerically the model (1a)-(1b). The flame position for a range of speed between 6 and $100 \mathrm{~cm} / \mathrm{s}$ is then shown in Fig. 2, where the position of the flame $z_{p o s}$ has been detected by calculating the axial position at which the average of the production term of temperature is maximum:

$z_{\text {pos }}=\max _{z}\left[\frac{1}{R} \int_{0}^{R} S_{T} d r\right]$.

Between 37.4 and $100 \mathrm{~cm} / \mathrm{s}$, the flame appears to be steady. Decreasing the speed just at $36.8 \mathrm{~cm} / \mathrm{s}$, the flame starts to oscillate and its average position is reported in Fig 2. Between 36.8 and $9.9 \mathrm{~cm} / \mathrm{s}$ a flame with repetitive extinction and ignition appears (FREI). In Fig. 2 the ignition and the extinction zone are represented. In the last range of speed, under $9.9 \mathrm{~cm} / \mathrm{s}$, a new stable flame appears according to the experiments [18]. This second

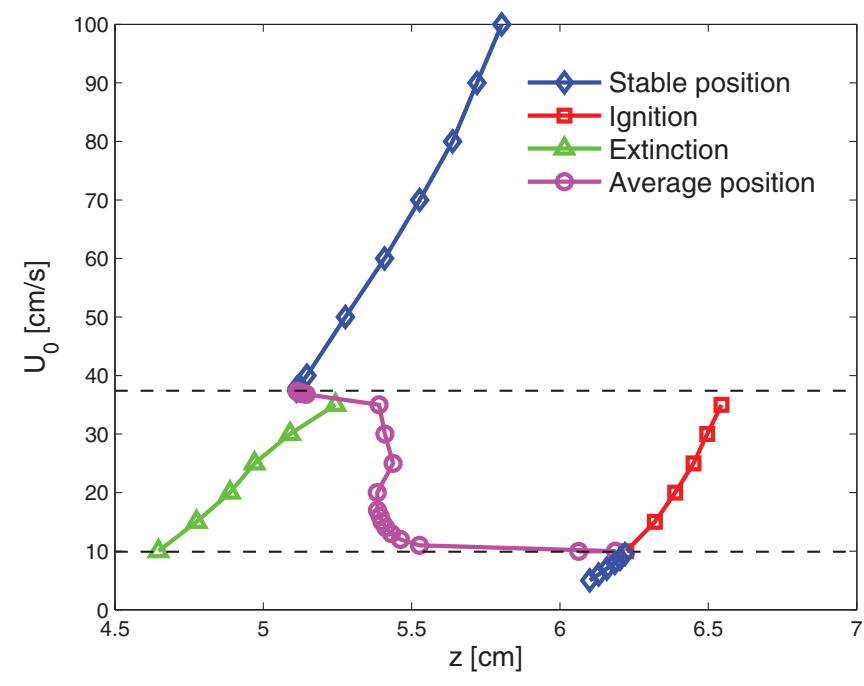

Fig. 2. Positions obtained with a moving mesh that can be compared with [14] $\left(D=0.6667 \mathrm{~cm}^{2} / \mathrm{s}, \quad A^{*}=1.455 \times 10^{9} \mathrm{~s}^{-1}\right)$. The two horizontal dashed lines mark the unstable region. $(\diamond)$ Position at which stabilization of flame occurs. ( $\square$ ) Position at which ignition occurs. $(\triangle)$ Position at which extinction occurs. $(\bigcirc)$ Average position of the unsteady flame.
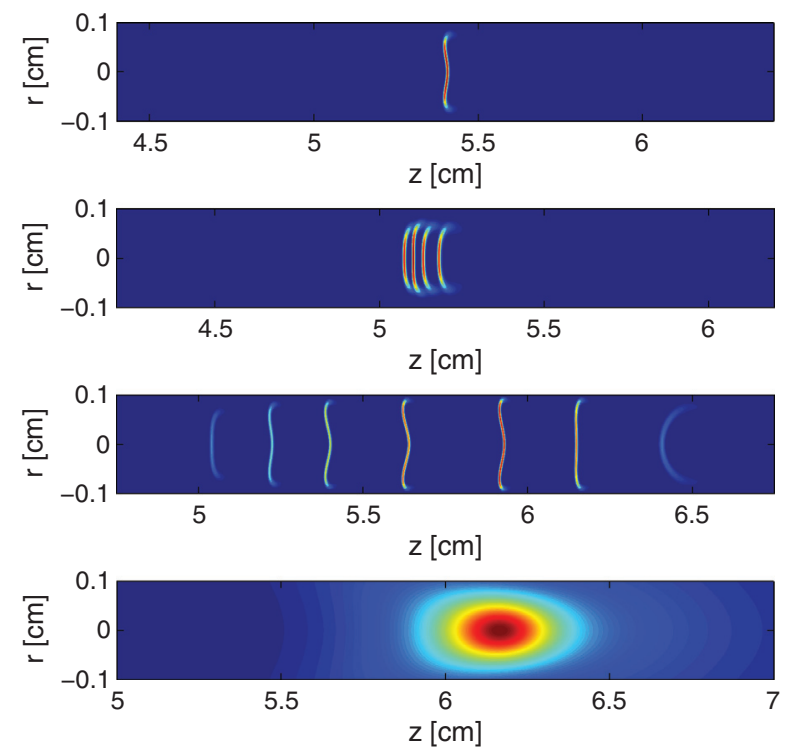

Fig. 3. Superposition of temperature production $S_{T}$ field in time. From top to bottom temperature production field of the steady flame $(60 \mathrm{~cm} / \mathrm{s})$, oscillating flame $(37 \mathrm{~cm} / \mathrm{s})$, FREI condition $(35 \mathrm{~cm} / \mathrm{s})$ and weak flame $(9 \mathrm{~cm} / \mathrm{s})$ are represented.

steady regime is called weak flame. It is possible to observe that, decreasing $U_{0}$, the average position of the flame in the FREI condition moves from the extinction point to ignition one. That means that near the upper bifurcation point, the flame moves fast toward the extinction point, once ignited. On the other hand, near the bottom stability threshold, the flame stays long time near the ignition point before moving to the extinction point. For a visual representation of the different behaviors encountered, in Fig. 3, the superposition of the production term of the same flame $S_{T}$ at different times is shown, for the different regimes found.

The numerical shapes for the two stable configurations are shown in Fig. 4. The temperature fields are obtained for $U_{0}=$ $40 \mathrm{~cm} / \mathrm{s}$, Fig. 4(a), and for $U_{0}=9.3 \mathrm{~cm} / \mathrm{s}$, Fig. 4(b). In the first case, Fig. 4(a), the maximum of temperature is $T=2730 \mathrm{~K}$. Strong gradients of temperature and concentration are present due to the flat flame front. On the other hand, for the weak stable flame, Fig. 4(b), 

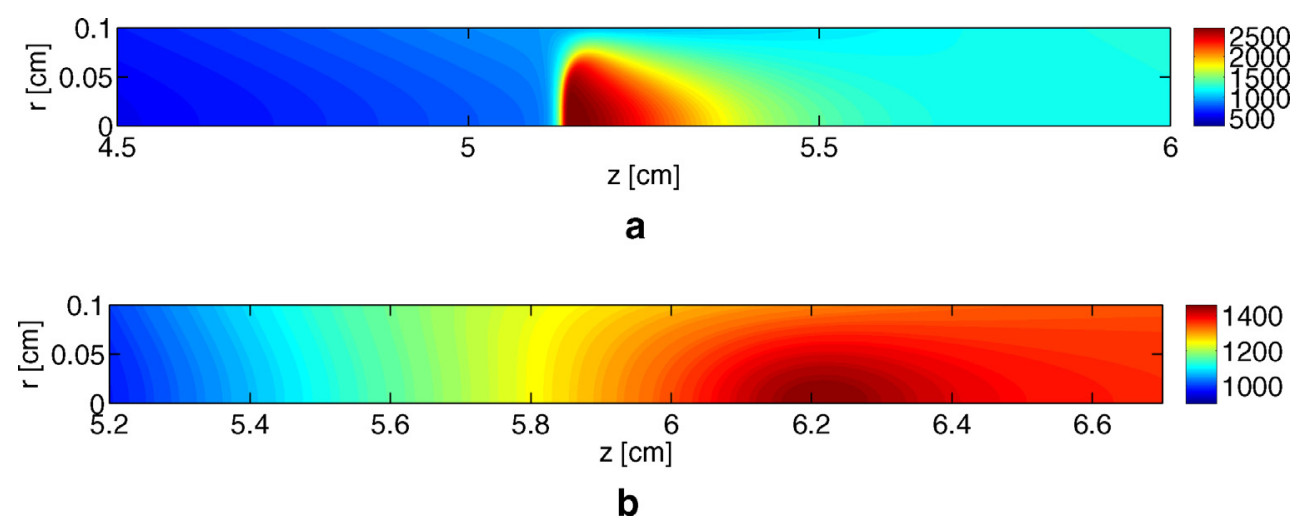

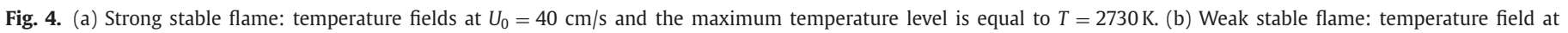
$U_{0}=9.3 \mathrm{~cm} / \mathrm{s}$ and maximum temperature level $T=1465 \mathrm{~K}$.

the maximum of temperature is slightly higher than the maximum of $T_{w}$. In this second case the flame appears to be diffused and it occupies such a large region that temperature and mass fraction gradients become weak. In both cases, the general character of the flame in the two steady conditions match the experiments [18]. The solutions in these two stable regions are fixed points in the phase diagram of the system. To understand the change of the state, near the two stability thresholds, the stationary solution has to be found for the mixture velocity $U_{0}$ at which the unstable behavior appears. This stationary solution is called basic state. In order to perform the stability analysis we linearize the system (1a)-(1b) around the basic state; the asymptotic behavior due to an infinitesimal perturbation then can be studied. Even if the system (1a)-(1b) is 3D, it is possible to make the hypothesis of homogeneity along the azimuthal direction because the basic state is invariant in the $\theta$-direction. Moreover this basic state is the solution that maximizes the symmetries, by definition. In this way the basic field is axisymmetric and inhomogeneous in $r$ and $z$ directions.

\subsection{Basic state}

The basic state is the solution of the steady problem. The stability analysis is performed around this state. When the dynamics is stable, the full system converges naturally to a steady solution which is also the basic state, as shown in the previous section. However, to characterize the instability bifurcations, we must investigate the unstable region. In this case, given that the full system does not converge to a steady state, its standard numerical simulation is not sufficient. Therefore, to compute the basic state outside the stable region, we have used the selective frequencies damping (SFD) method [27,28]. The objective of this method is to eliminate the characteristic frequencies of the instability and thus prolonging the steady state within a supercritical regime (when this solution is linearly unstable). This method has the great advantage of being easily implemented regardless of the equation or problem to solve. If $\mathbf{q}$ is the vector of variables $\mathbf{q}=(Y, T)^{t}$ and $\dot{\mathbf{q}}$ is the temporal derivative, the system (1) can be written $\dot{\mathbf{q}}=f(\mathbf{q})$ and the filtered system is as follows:

$$
\left\{\begin{array}{l}
\dot{\mathbf{q}}=f(\mathbf{q})-\chi(\mathbf{q}-\overline{\mathbf{q}}) \\
\dot{\overline{\mathbf{q}}}=\frac{\mathbf{q}-\overline{\mathbf{q}}}{\Delta}
\end{array}\right.
$$

where $\chi$ is a control parameter and $\Delta$ is the cut-off frequency. To avoid instabilities due to numerical noise, [26], the solution of the filtering operation is considered to be converged when a residual level of $10^{-10}$ is obtained. The basic states or the phasespace fixed points are shown in Fig. 5(a) and (b), respectively for the temperature and for the concentration for a flow with velocity $U_{0}=37 \mathrm{~cm} / \mathrm{s}$. The fixed points for a flow velocity $U_{0}=9.955 \mathrm{~cm} / \mathrm{s}$ are shown in Fig. 6(a) and (b), near the bifurcation point corresponding to a weak flame.

\subsection{Linearized dynamics and stability analysis}

In this section the linear stability of this basic state $\mathbf{Q}_{b}=$ $\left(Y_{b}, T_{b}\right)^{t}$ is studied. The proposed global linear stability analysis is based on the classical perturbation technique where the instantaneous flow $\mathbf{q}$ is the composition of the basic state and an unknown perturbation $\mathbf{q}^{\prime}=\left(Y^{\prime}, T^{\prime}\right)^{t}$ :

$\mathbf{q}(r, \theta, z, t)=\mathbf{Q}_{b}(r, z)+\varepsilon \mathbf{q}^{\prime}(r, \theta, z, t)$

with $\varepsilon \ll 1$. Decomposition (9) is introduced into Eqs. (1a) and (1b). The resulting equations are then simplified, first by taking into account that the basic quantities satisfy the governing equations and secondly by assuming that the fluctuating quantities are small, so that these equations can be linearized with respect to the disturbance. The equations for the perturbation can be eventually written as

$$
\begin{aligned}
& \frac{\partial Y^{\prime}}{\partial t}+U_{z} \frac{\partial Y^{\prime}}{\partial z}=D\left[\frac{1}{r} \frac{\partial Y^{\prime}}{\partial r}+\frac{\partial^{2} Y^{\prime}}{\partial r^{2}}+\frac{1}{r^{2}} \frac{\partial^{2} Y^{\prime}}{\partial \theta^{2}}+\frac{\partial^{2} Y^{\prime}}{\partial z^{2}}\right]+ \\
& -A e^{\left(-\frac{T_{a}}{T_{b}}\right)} Y^{\prime}-A Y_{b} e^{\left(-\frac{T_{a}}{T_{b}}\right)}\left[\frac{T_{a}}{T_{b}^{2}}\right] T^{\prime} \\
& \frac{\partial T^{\prime}}{\partial t}+U_{z} \frac{\partial T^{\prime}}{\partial z}=D\left[\frac{1}{r} \frac{\partial T^{\prime}}{\partial r}+\frac{\partial^{2} T^{\prime}}{\partial r^{2}}+\frac{1}{r^{2}} \frac{\partial^{2} T^{\prime}}{\partial \theta^{2}}+\frac{\partial^{2} T^{\prime}}{\partial z^{2}}\right]+ \\
& +\frac{Q}{C_{p}} A e^{\left(-\frac{T_{a}}{T_{b}}\right)} Y^{\prime}+\frac{Q}{C_{p}} A Y_{b} e^{\left(-\frac{T_{a}}{T_{b}}\right)}\left[\frac{T_{a}}{T_{b}^{2}}\right] T^{\prime}
\end{aligned}
$$

Since the basic state is stationary, the solutions of the linearized equation are autonomous, so it is possible to write the perturbation as a normal mode form (Fourier-Laplace transform):

$\mathbf{q}^{\prime}(r, \theta, z, t)=\hat{\mathbf{q}}(r, z ; m, \lambda) e^{i m \theta+\lambda t}+$ c.c.

The circular frequency $\lambda$ is a complex number: its real part $\lambda_{r}$ represents a temporal growth rate and its imaginary part $\lambda_{i}$ the frequency of the perturbation. The physical meaning of $\lambda_{r}$ is in accordance with the classical definition of stability: for $\lambda_{r}>0$, the basic state is unstable whereas for $\lambda_{r}<0$ the basic state is stable. $m \in \mathbb{Z}$ is the azimuthal wave number and $\hat{\mathbf{q}}(r, z)=(\hat{Y}(r, z), \hat{T}(r, z))^{t}$ is the complex eigenfunction vector associated with the eigenvalue $\lambda$. c.c. represents the complex conjugate. Mathematical form (11) is 


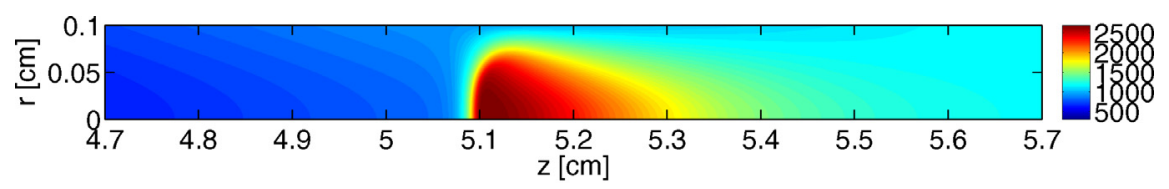

a

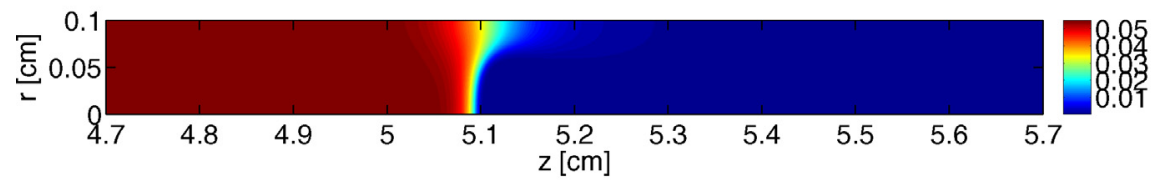

b

Fig. 5. Basic state for the temperature field (a) and for the concentration field (b) obtained by the SFD method. Average speed of fluid is equal to $37 \mathrm{~cm} / \mathrm{s}$.

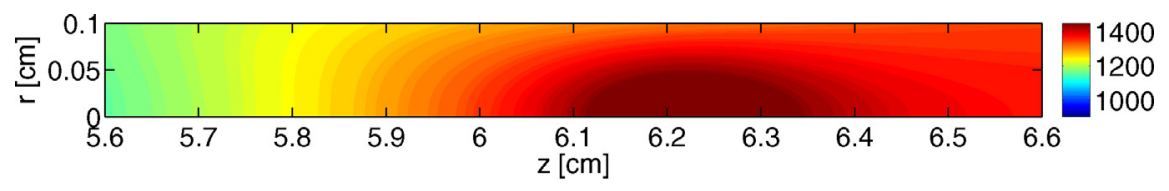

a

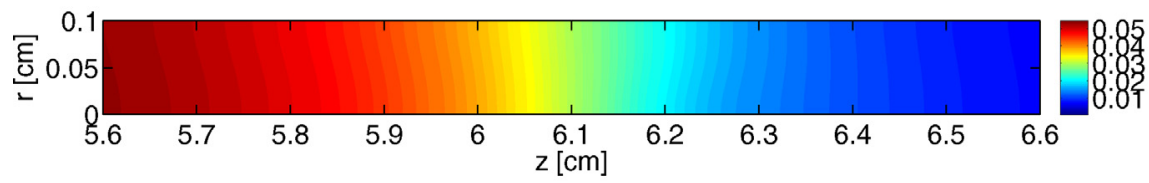

b

Fig. 6. Basic state for the temperature field (a) and for the concentration field (b) near the weak flame threshold. Average speed of fluid is equal to $9.955 \mathrm{~cm} / \mathrm{s}$.

then introduced into Eqs. (10a) and (10b), the linearized equations become an eigenvalue problem:

$$
\left(\left[\begin{array}{cc}
\mathcal{L}_{Y}\left(T_{b}, m\right) & -\mathcal{P}_{T}\left(Y_{b}, T_{b}\right) \\
\mathcal{Q} \mathcal{P}_{Y}\left(T_{b}\right) / C_{p} & \mathcal{L}_{T}\left(Y_{b}, T_{b}, m\right)
\end{array}\right]-\lambda I\right)\left[\begin{array}{l}
\hat{Y} \\
\hat{T}
\end{array}\right]=0,
$$

where $\mathcal{L}_{Y}$ is the linear operator acting on $\hat{Y}$ in Eq. (10a), $\mathcal{P}_{T}$ the production operator acting on $\hat{T}$ in Eq. $(10 \mathrm{a}), \mathcal{L}_{T}$ is the linear operator acting on $\hat{T}$ in Eq. (10b) and $\mathcal{P}_{Y}$ the production operator acting on $\hat{Y}$ in Eq. (10b). $\mathcal{L}_{Y}, \mathcal{P}_{T}, \mathcal{L}_{T}$ and $\mathcal{P}_{Y}$ are given in Appendix A. The resulting linear operator depends on the basic state $\mathbf{Q}_{b}$ and the azimuthal wave number $m$. The matrix in Eq. (12) must respect the following boundary conditions for the perturbed problem:

- Inflow condition at $z=0$ :

$$
\hat{T}(r, 0)=0, \quad \hat{Y}(r, 0)=0
$$

- Outflow condition at $z=L$ :

$$
\frac{\partial \hat{T}(r, L)}{\partial z}=0, \quad \frac{\partial \hat{Y}(r, L)}{\partial z}=0
$$

- Symmetric condition is applied at channel axis at $r=0$ :

$$
\frac{\partial \hat{T}(0, z)}{\partial r}=0, \quad \frac{\partial \hat{Y}(0, z)}{\partial r}=0
$$

- At channel wall $r=R$

$$
\hat{T}(R, z)=0, \quad \frac{\partial \hat{Y}(R, z)}{\partial r}=0
$$

The boundary conditions are consistent with the time evolution of the system (1), since the solution of the direct numerical simulation can be approximated with the sum of the basic state and a perturbation.

From a numerical point of view, the discretized operator of linear system (12) has been built by blocks. This has been done involving the Kroneker product between the discretized operator and the identity matrix $\mathbf{D} \otimes \mathbf{I}$ and vice versa $\mathbf{I} \otimes \mathbf{D}$ depending on the direction in which the operator is applied $[29,30]$. The eigenvalue problem has been then solved using Arnoldi's method, having imposed boundary conditions. We have looked for the first 100 modes with the largest real part, taking a residual level of Arnoldi's algorithm equal to $10^{-7}$. More information about the numerical convergence is provided in Appendix B.

\section{Numerical simulations of the model}

\subsection{Flame dynamics}

As shown in Fig. 2, the FREI regime exists between two stable zones. The first stability threshold is at $37.4 \mathrm{~cm} / \mathrm{s}$ and the second is at about $10 \mathrm{~cm} / \mathrm{s}$. Moreover, in correspondence with the first threshold the flame becomes unsteady. This is noticed looking at the average position in Fig. 2. This state modification is characterized by a bifurcation when the equilibrium solution becomes unstable. In this section, the two bifurcation points are studied by letting evolve in time the system (1a)-(1b). We want to point out the physical mechanisms underlying the unsteady dynamics of the model. For the first stability threshold, starting with a fluid velocity for which the flame is steady, the flow rate of methane is gradually reduced, and the flame position is observed. Decreasing the flow rate of mixture, the flame starts oscillating and the oscillation amplitude is linked to the flow speed value $U_{0}$. In Fig. $7, z_{\text {pos }}$ is showed as a function of time. The flame position was interpolated with a 7th-order polynomial to avoid spurious effects due to the $z$-discretization. From the pulsating behavior of the flame, it is possible to infer the amplitude $|A|$ (17) of the limit-cycle in the region between the stable condition and the FREI one,

$|A|=\left|z_{\text {pos }}^{\max }-z_{\text {pos }}^{\min }\right|$ 


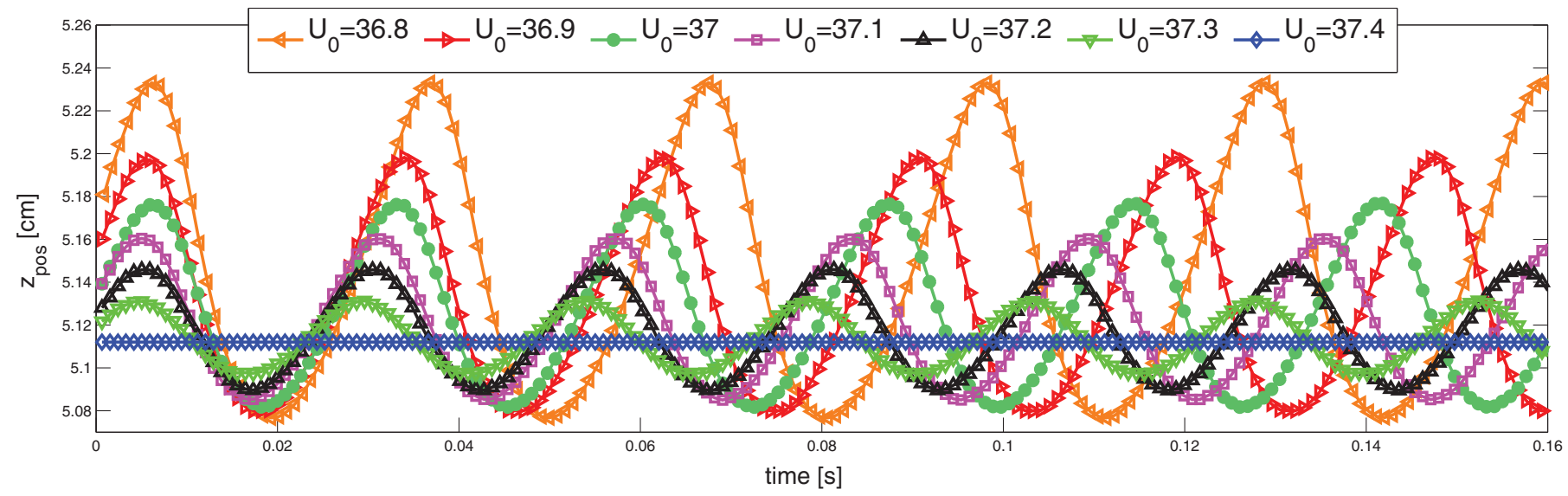

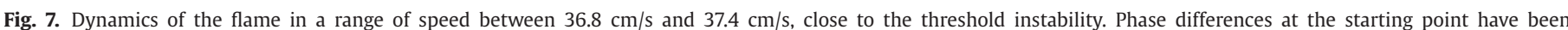
eliminated by a shift. Decreasing the flow speed, the oscillation amplitude increases, while the oscillation frequency increases with increasing the flow speed.

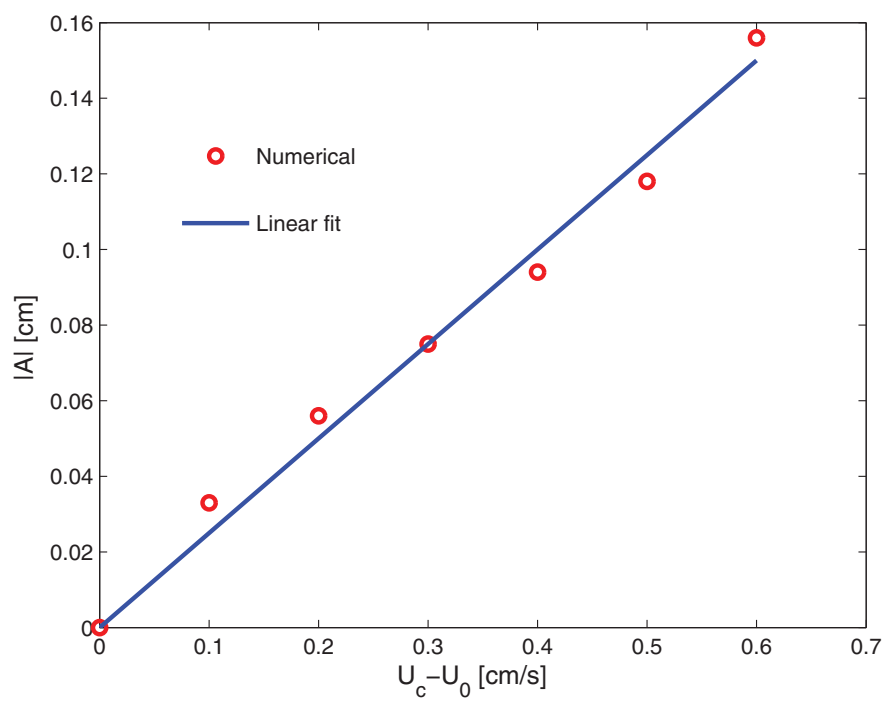

Fig. 8. Oscillation amplitude $|A|$ of the flame position as a function of $U_{c}-U_{0}$, with $U_{c}=37.4 \mathrm{~cm} / \mathrm{s}$.

As shown in Fig. 8, for all the speeds in this range the flame oscillates and the amplitude increases with decreasing the flow speed $U_{0}$. Figure 8 shows a continuous growth of the amplitude, without jumps in the transition from stable to unstable conditions. Moreover, the amplitude appears to increase linearly $|A| \sim$ $0.5 \times\left(U_{c}-U_{0}\right)$ where $U_{c}$ is the critical speed at which the system become unstable. $U_{c}$ is set equal to $37.4 \mathrm{~cm} / \mathrm{s}$, see Fig. 7. The diagram in Fig. 8 excludes the presence of any subcritical bifurcation but it cannot explain the kind of bifurcation as $z_{\text {pos }}$ is not a state variable. For this reason, temperature and concentration have been recorded in a point within the range of position where the flame oscillates. The resulting limit cycle is reported in Fig. 9. It is possible to note that decreasing the mixture velocity from $37.4 \mathrm{~cm} / \mathrm{s}$ to $36.8 \mathrm{~cm} / \mathrm{s}$ the limit cycle increases its dimension. Projecting the limit cycle on the $x$ and $y$ axes it is possible to obtain the amplitude of the temperature $|T|$ and concentration $|Y|$, see Fig. 10. It is possible to observe that decreasing the speed, both amplitudes increase proportionally to $\sqrt[3]{U_{0}}$. Hence, it turns out that the transition is given by a supercritical Hopf bifurcation, as no jump and no hysteretic behavior are present.

Let us now analyze the integral values of all terms in Eqs. (1a) and (1b), in the whole domain. Specifically, the integral of advection, radial diffusion, axial diffusion and the production terms

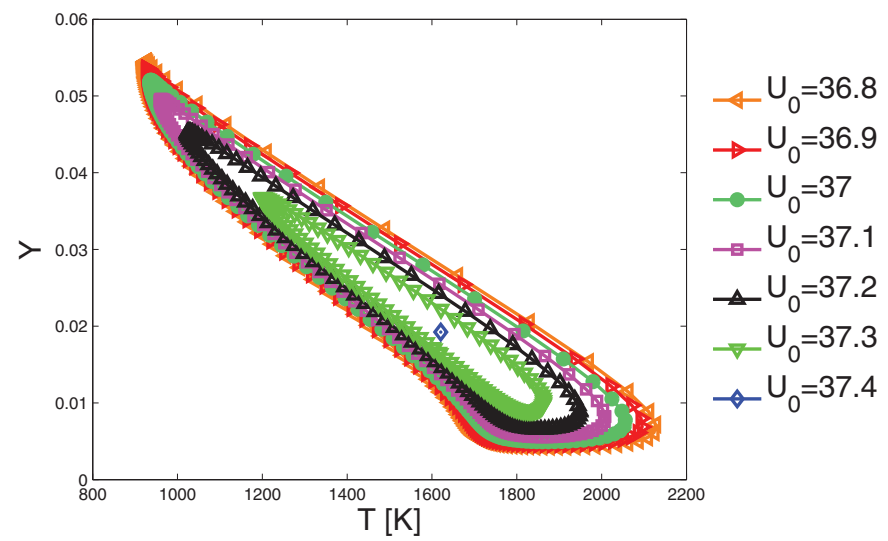

Fig. 9. Limit cycle in a range of $U_{0}$ from 36.8 to $37.4 \mathrm{~cm} / \mathrm{s}$.

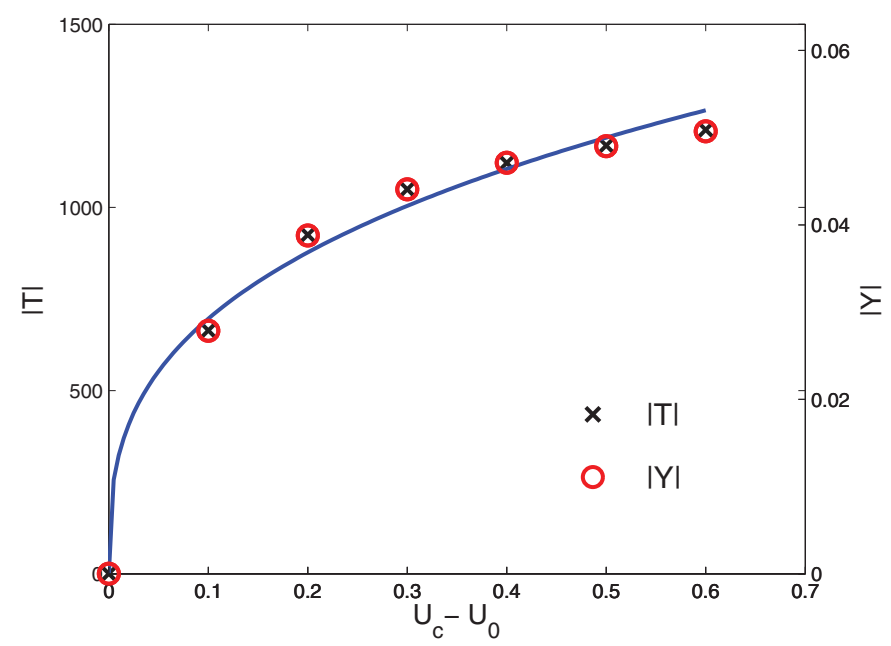

Fig. 10. Amplitude of temperature $|T|(\times)$ and concentration $|Y|(\bigcirc)$ versus $U_{c}-U_{0}$.

are evaluated and plotted as a function of time in Fig. 11(a) and (c), for a speed flow $U_{0}=37 \mathrm{~cm} / \mathrm{s}$. The integral values of Eq. (1a) are linked to the trend of the mass fraction contained in the numerical domain, instead the integral value of the terms in Eq. (1b) are linked to the energy within the domain, proportional to the multiplicative constant of the specific heat. In Fig. 11 for the 


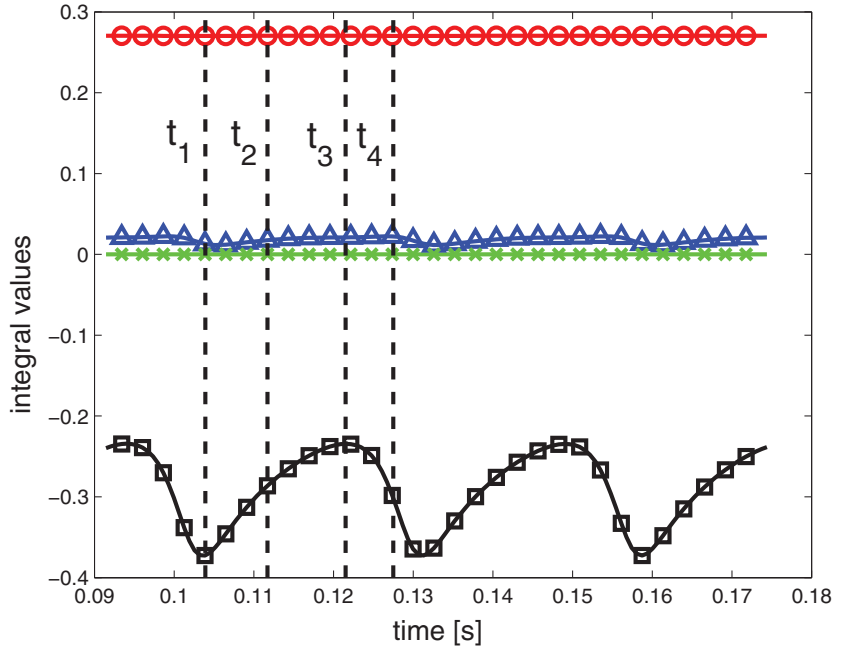

a

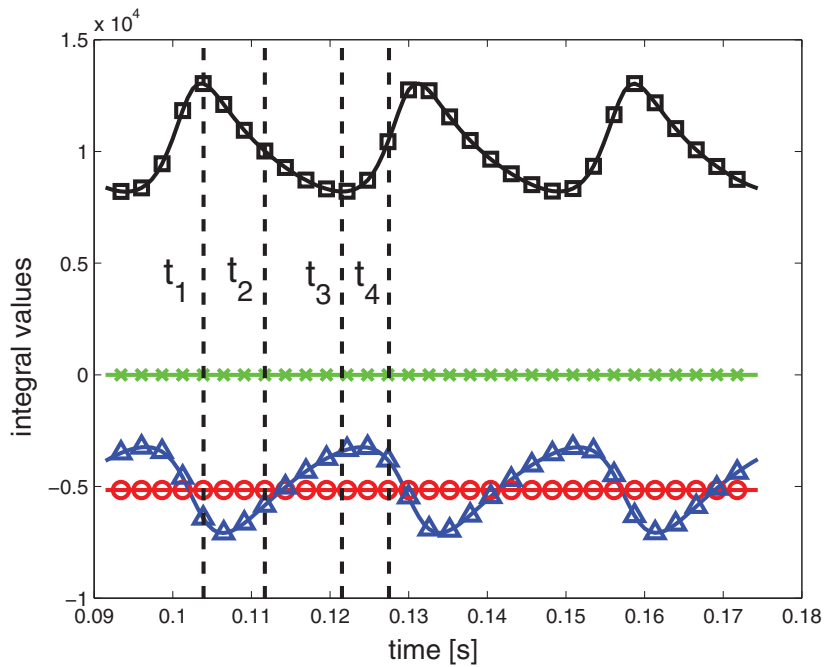

C

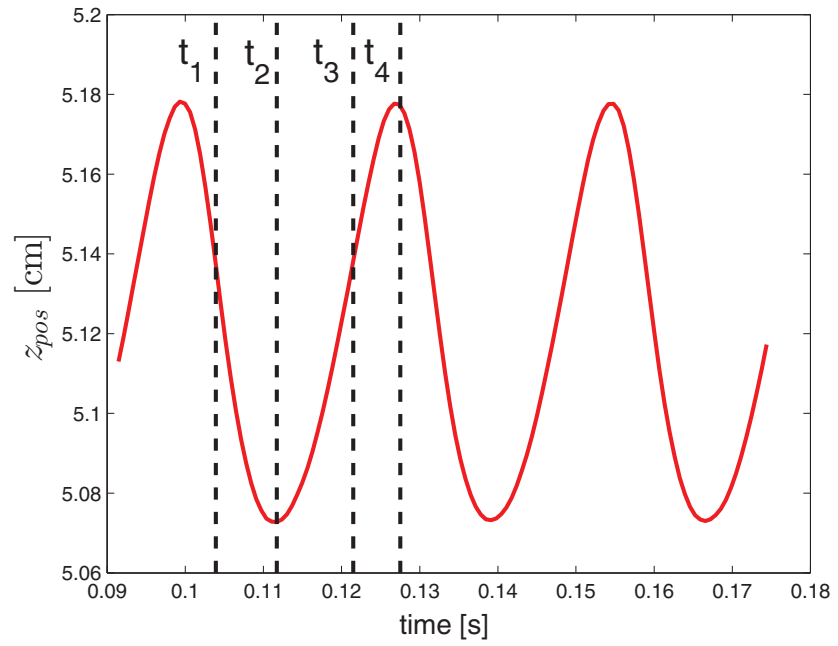

e

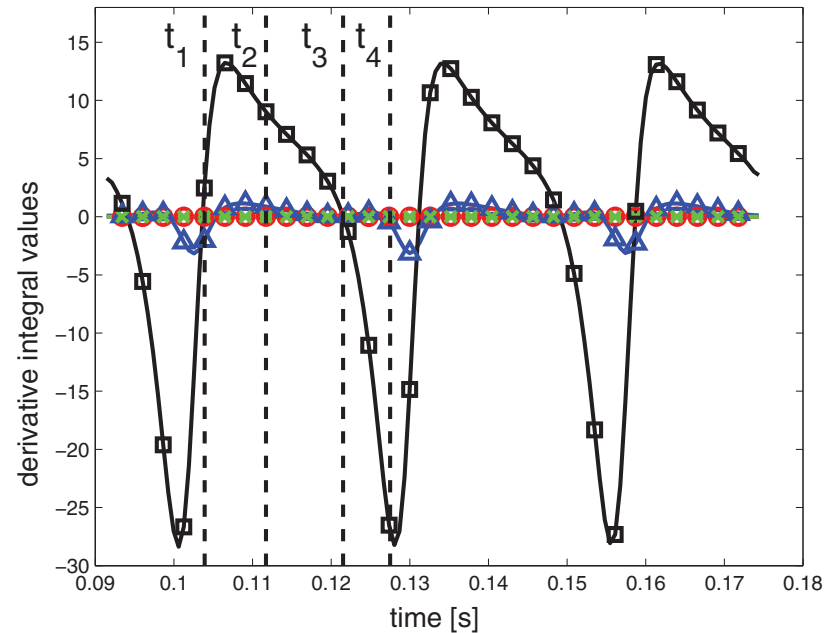

b

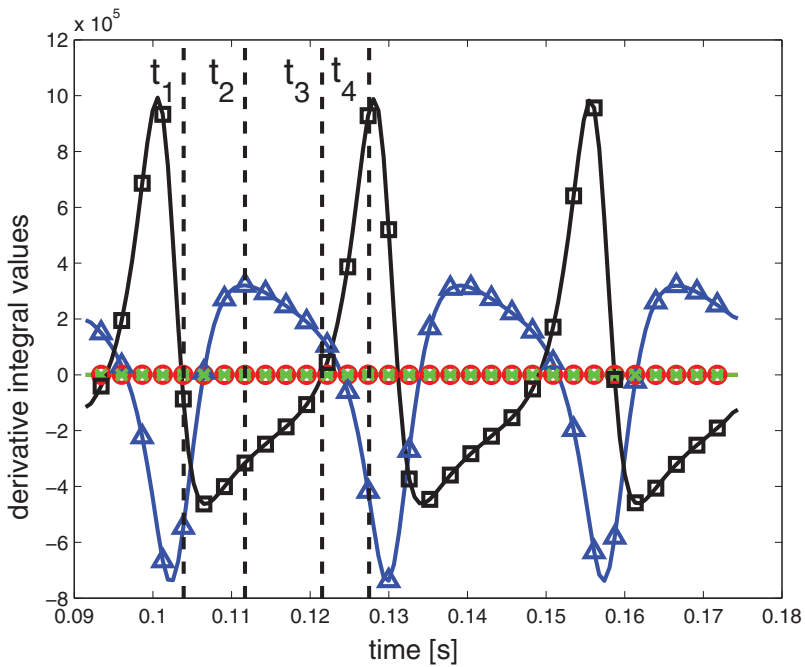

d

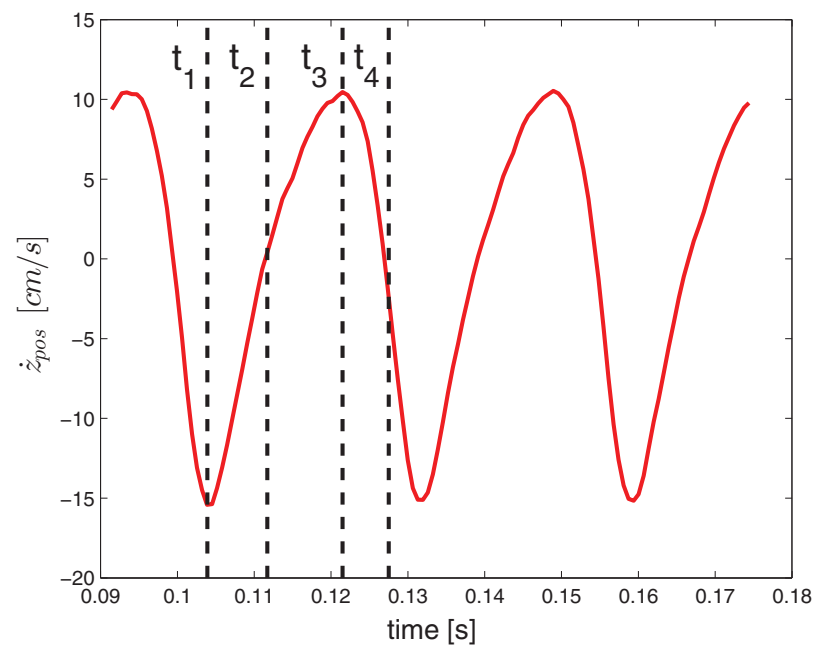

f

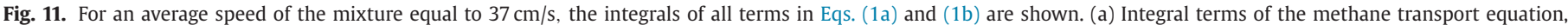

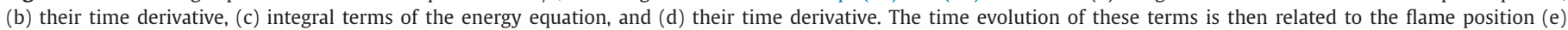

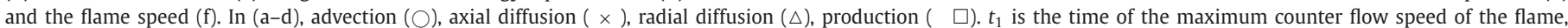
$t_{2}$ time of minimum flame position, $t_{3}$ time of maximum flame speed and $t_{4}$ time of maximum flame position. 


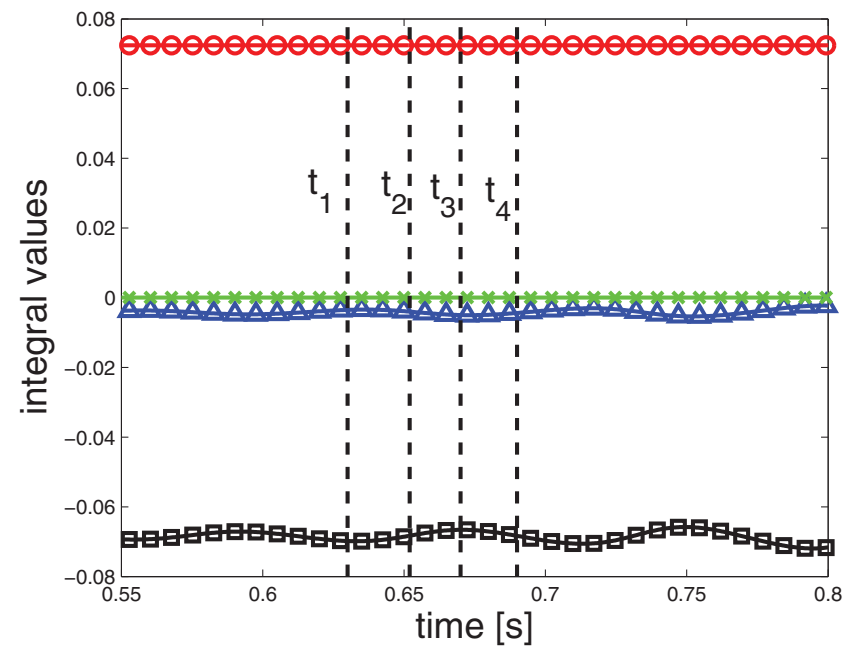

a

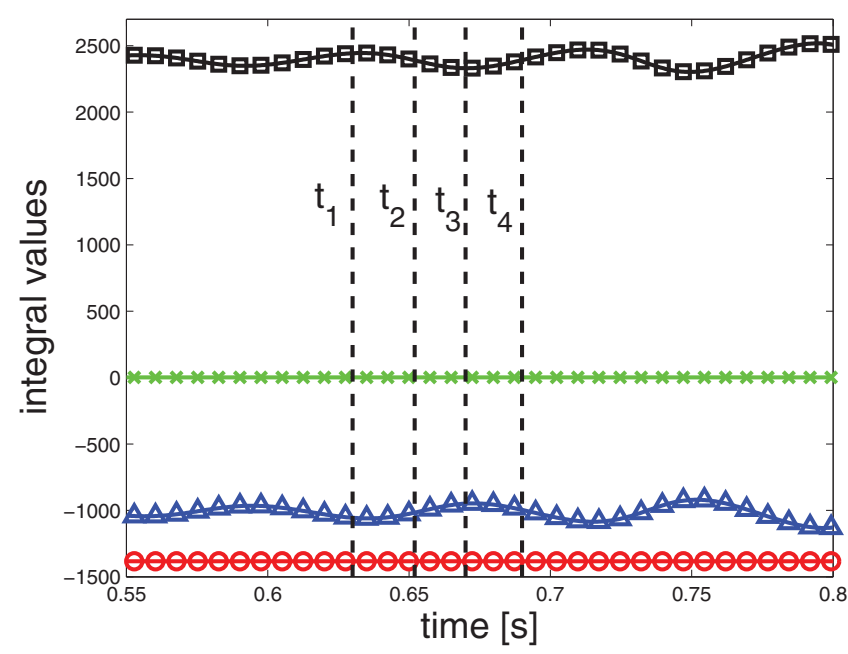

C

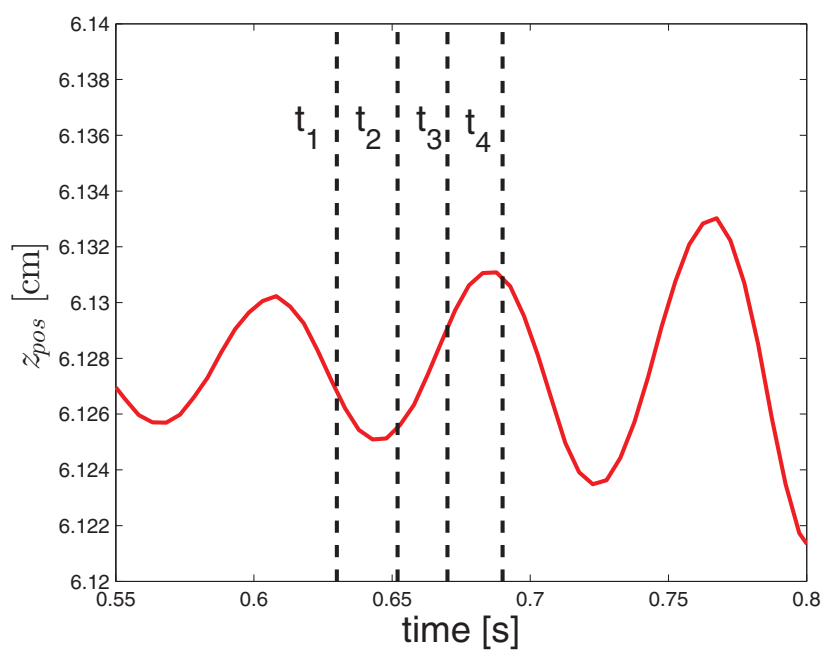

e

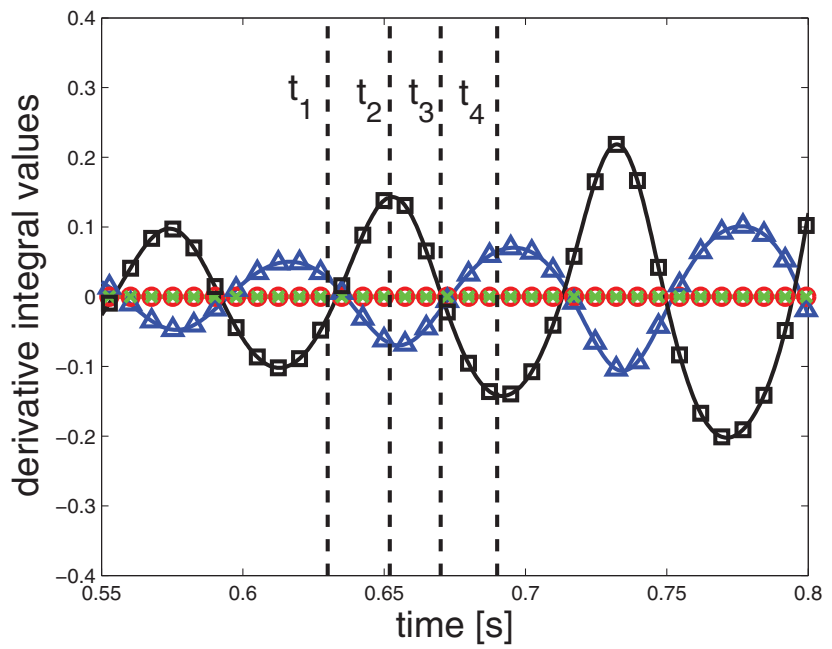

b

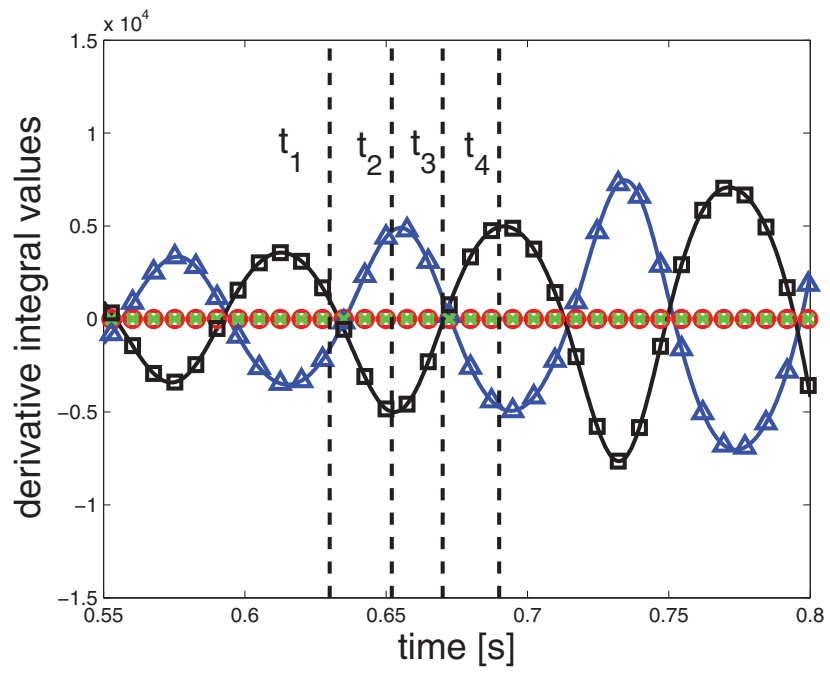

d

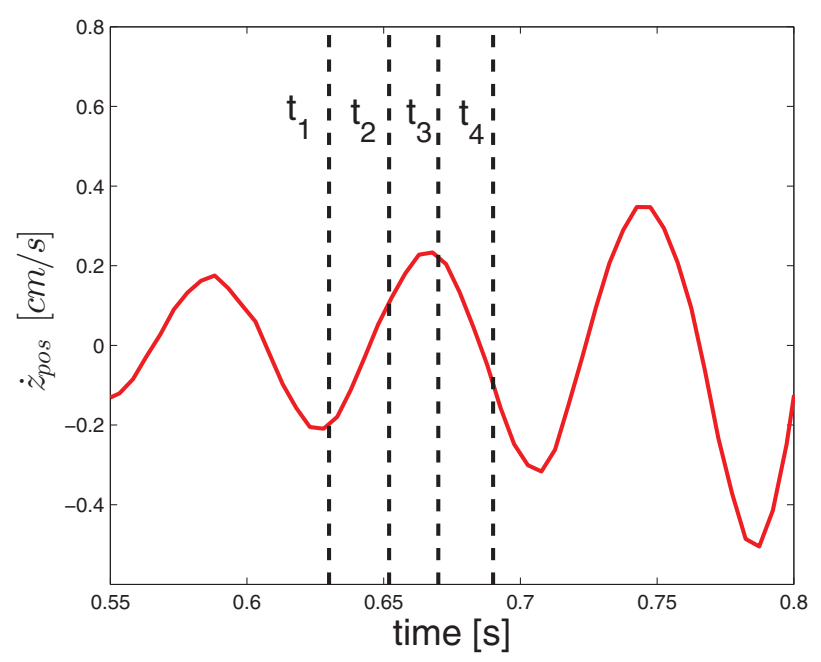

f

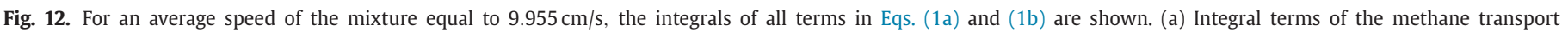

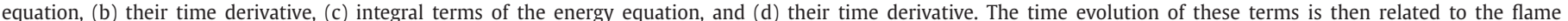

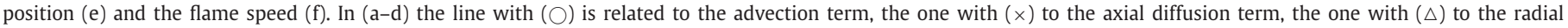

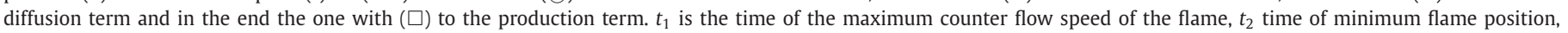
$t_{3}$ time of maximum flame speed and $t_{4}$ time of maximum flame position. 


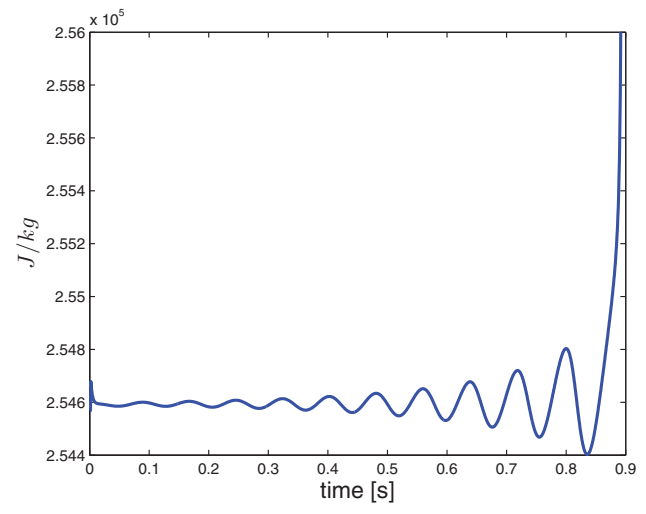

a

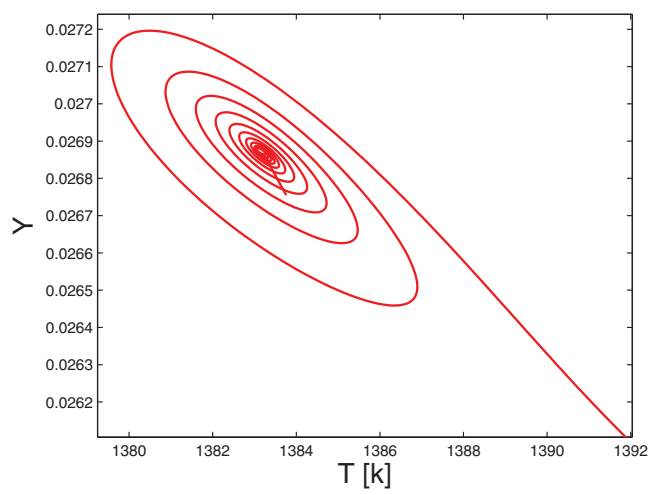

b

Fig. 13. (a) Internal energy trend and (b) phase diagram at $9.955 \mathrm{~cm} / \mathrm{s}$ initializing the simulation with the steady solution obtained at $9.95 \mathrm{~cm} / \mathrm{s}$.

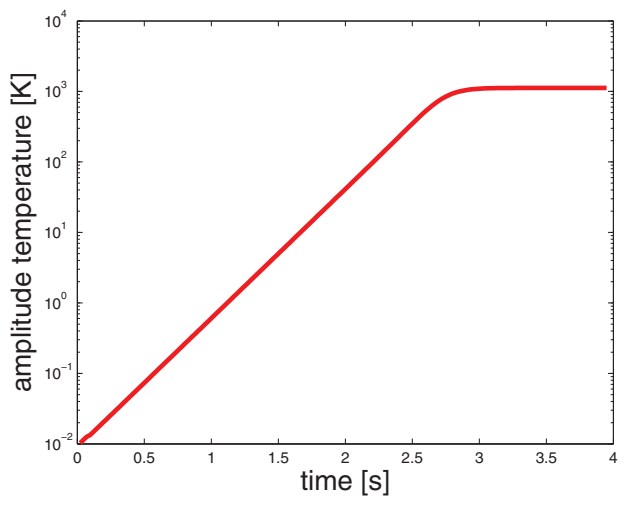

a

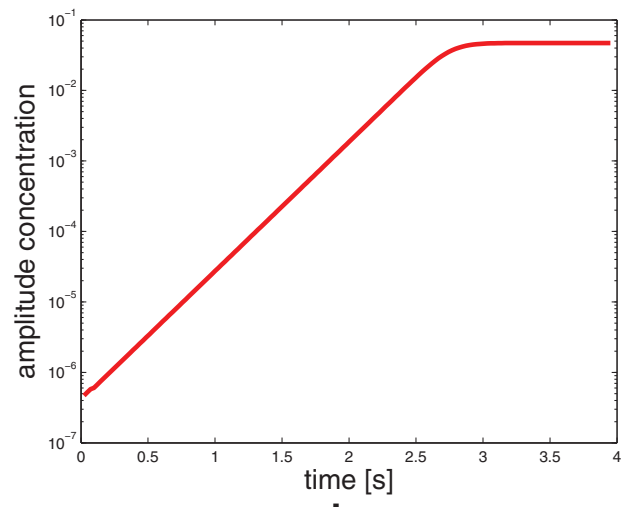

b

Fig. 14. Growth rate of temperature (a) and concentration (b). The evolution is linear up to the non-linear saturation. The slope of the curve is $\lambda_{r}=4.2 \mathrm{~s}^{-1}$.

integral values of all terms of Eqs. (1a) and (1b) and for the position of the flame there are also their time derivative. The time evolution of the production terms (Fig. 11(a) and (c)) are in phase opposition, as it can be inferred directly from Eqs. (1a) and (1b). From Fig. 11(a), we can see that the instability dynamics is not driven by the mass fraction equation, since the advection is able to compensate the fuel-consumption. The evolution of energy provides the necessary information for the understanding of the oscillating behavior. Figure 11(c) shows that the radial diffusion is in opposition with the reaction. To explain this behavior we can refer to the time-scales of the two phenomena at play. The reaction time scale is proportional to $A^{-1}$, whereas the diffusion is proportional to $h^{2} / D$ where $h$ is a characteristic length. Therefore, the typical chemical time-scale is much smaller than the diffusive one. We explain the resulting dynamics in the following way: if the speed flame is high-enough then the diffusion is able to equilibrate the reactive term, otherwise the oscillating behavior appears. Since the compressibility effects are not taken into account, the flame speed turns out to be an approximation of the vectorial sum between the reaction speed (counterflow direction) and the mixture one $U_{0}$. The reaction speed is equal to the speed the flame would have if $U_{0}=0 \mathrm{~cm} / \mathrm{s}$ and the channel was completely filled with fresh mixture at $t=0 \mathrm{~s}$. With this in mind, we can give the following picture: when the reaction term reaches its peak ( $t_{1}$ in Fig.11(c), and (d)) the flame attains the maximum speed in the counterflow direction, Fig. 11(f). After this point, the reaction term decreases as the radial diffusivity increases. The flame stops $\left(t_{2}\right.$ in Fig. 11(e)) because it is in a region where the wall temperature is low, and at this point $\left(t_{2}\right)$ the diffusion growth is maximum, Fig. 11(d). The sustainability of the combustion is ensured by the fact that the flame moves itself in the flow direction where the wall temperature is higher and the diffusion growth slows down. Again the maximum value of the flame speed is linked to a zero derivative of the reaction (Fig. 11(d) in $t_{3}$ time value). After the minimum value of the reaction, the flame speed increases again, because of the higher temperature at wall. The flame stops at $t_{4}$, Fig. 11(e), where there is the maximum reaction growth and the radial diffusion strongly decreases, see Fig. 11(c) and (d). At this point, the flame reverses its velocity to enter the zone richer of combustible and the loop starts again.

The same analysis can be carried out for the weak flame. Starting from a steady solution in the weak flame region, for instance at $9.95 \mathrm{~cm} / \mathrm{s}$, and then increasing the velocity crossing the FREI threshold, for example at $9.955 \mathrm{~cm} / \mathrm{s}$, the solution evolves towards the FREI condition passing through an oscillating behavior that grows exponentially. The integral values of advection, radial diffusion, axial diffusion and production for both temperature and concentration fields have been investigated during the transient regime. The results are shown in Fig. 12. The mechanism underlying the FREI regime is still the counter phase shift between the production and the radial diffusion. As before, in Fig. $12, t_{1}, t_{2}, t_{3}$ and $t_{4}$ denote respectively the maximum integral value of production, the maximum growth of the radial diffusion, the minimum integral value of the production and the maximum growth of the production. When the FREI regime is reached, other mechanisms are involved and the integral values are not sufficient to describe the dynamics. Near the weak flame threshold, FREI regime might be affected by local dynamics or non-linear 


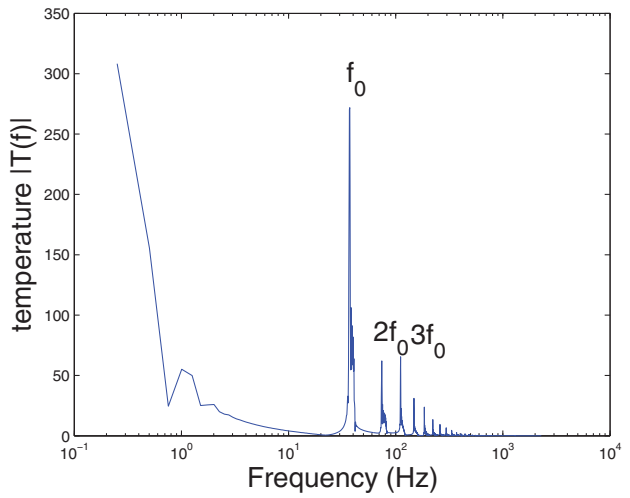

a

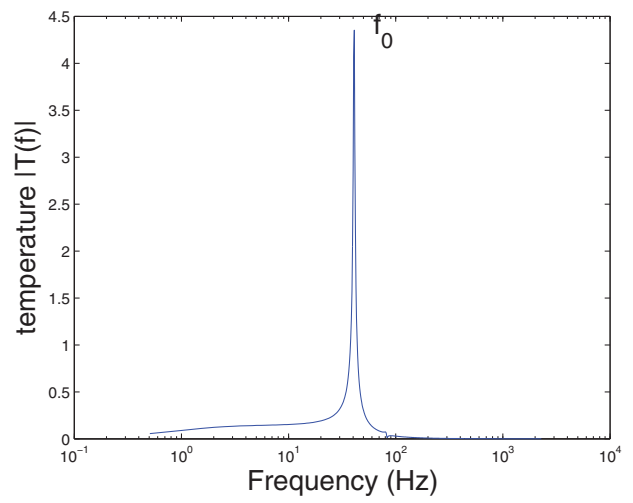

b

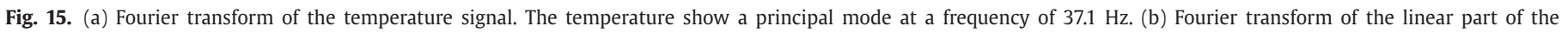
temperature signal. Concentration and temperature show only a mode at a frequency of $41 \mathrm{~Hz}$.

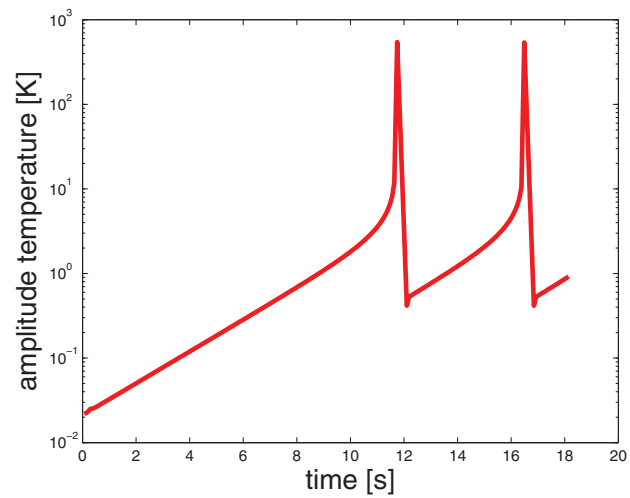

a

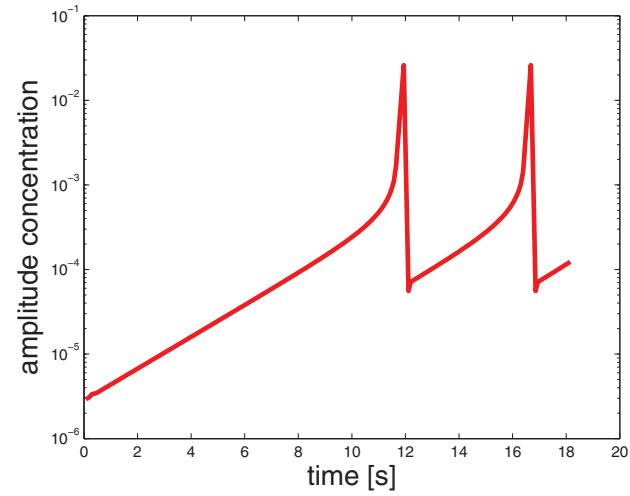

b

Fig. 16. Growth rate of temperature (a) and concentration (b). The evolution is perfectly linear up to non-linear saturation.

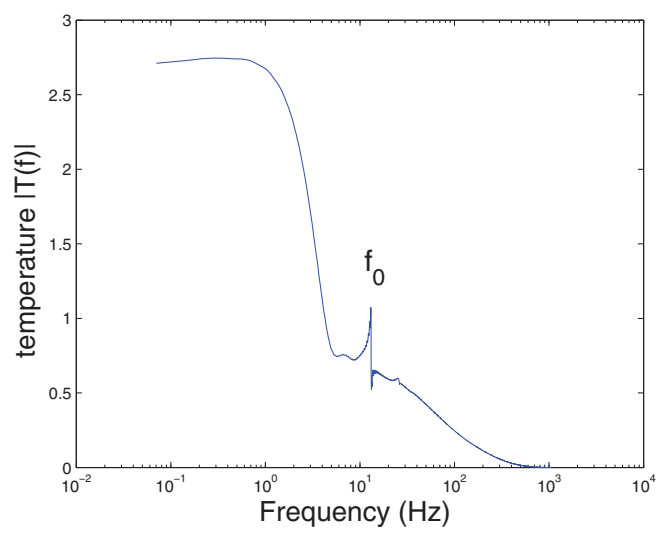

a

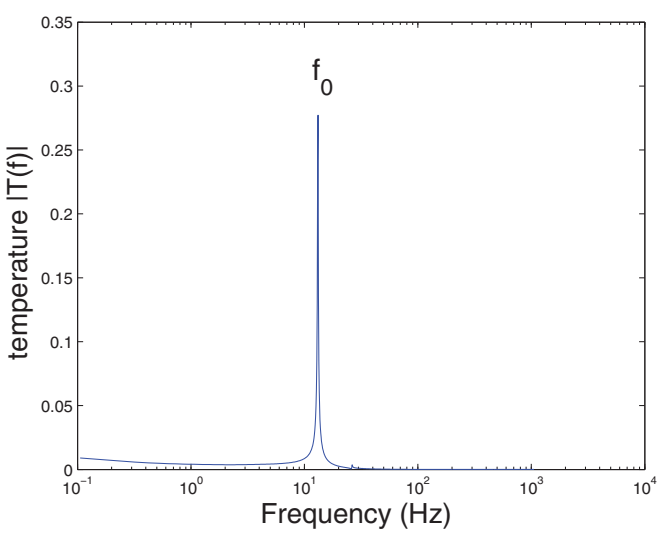

b

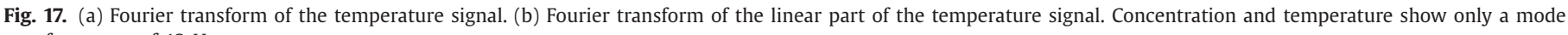
at a frequency of $13 \mathrm{~Hz}$.

interaction between the three mechanisms, convection, diffusion and reaction. Nonetheless, the source of the instability is global, and it depends on the contrast between loss and production of heat.

The transition is not only global but even linear. The instantaneous internal energy is provided by the sum of the internal energy of each chemical species assuming the specific heat $C_{v}$ constant with the temperature, see Fig. 13(a). First, the solution moves itself in the basin of attraction of the steady solution at $9.955 \mathrm{~cm} / \mathrm{s}$, then starts moving around it, see Fig. 13(b). At the same time, the energy of the perturbation starts growing exponentially, subtracting energy from the basic state. This behavior ensures that the evolution can be correctly described by a linear approximation at small times. This will be verified later by the linear stability analysis.

Figure 13 (a) suggests another information. Moving from $9.95 \mathrm{~cm} / \mathrm{s}$ to $9.955 \mathrm{~cm} / \mathrm{s}$, the stable solution is not prolonged in the FREI region but it becomes unstable, no hysteric behavior is 


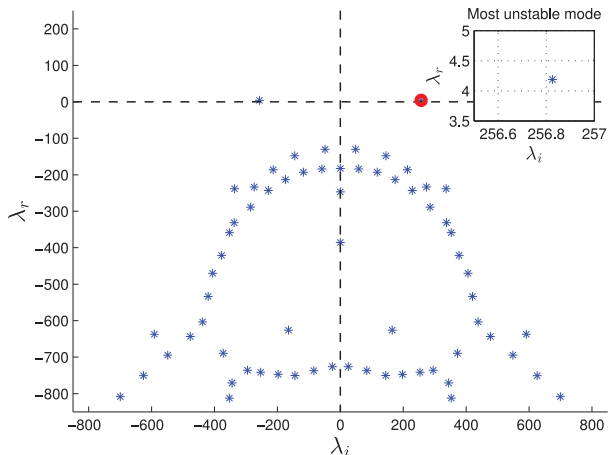

Fig. 18. Spectrum of the 100 most unstable modes. Speed flow is equal to $37 \mathrm{~cm} / \mathrm{s}$. In the inset, the unstable mode is highlighted.

observed. Therefore, this second transition also turns out to be a supercritical Hopf bifurcation.

\subsection{Stability analysis by numerical simulation}

In order to characterize the instability, we initialize the simulation of the model with the basic state. Until the non-linear terms remain small, the time evolution can be described by a linear approximation of the model. The numerical simulation initialized with the basic state is perturbed by adding a noise in the temperature and concentration field. This is generated by summing a random field of values between 0 and 1 and multiplied by $10^{-7}$. This noise is sufficient to destabilize the basic state. In this way the behavior is driven by the most unstable mode that is hence triggered. The solution of the direct simulation moves away from the basic state. Plotting in a semi-logarithmic plane the amplitude of the temperature and concentration oscillations registered in a point close to the flame of the basic state, we can observe the growth rate of the unstable mode. In Fig. 14, the temporal evolution of the concentration and temperature amplitude are shown. For small times, the evolution is linear. After the linear regime, the non-linear terms are no longer negligible and amplitude saturates. Computed through a linear regression, the temporal growth rate is equal to $\lambda_{r}=4.2 \mathrm{~s}^{-1}$. The Fourier transform of the temperature signal can be used to compute the exact frequency. Figure 15(a) shows the Fourier spectrum of the temperature. The spectrum of the concentration reveals the same modes and thus it is not shown here. The spectrum exhibits one main frequency (the other peaks correspond to the harmonics) of $37.1 \mathrm{~Hz}$. However, it is worth underlining that Fig. 15(a) shows the spectrum of the signal with both the linear and non-linear perturbations, when the limit-cycle is reached. Considering only the linear growth range, the spectrum is markedly different, Fig. 15(b), with only one mode present. Its frequency is equal to $41 \mathrm{~Hz}$.

The same procedure has been carried out for the instability at low speed. The basic state, that was subsequently perturbed, has been found for a mixture velocity equal to $U_{0}=9.955 \mathrm{~cm} / \mathrm{s}$. A

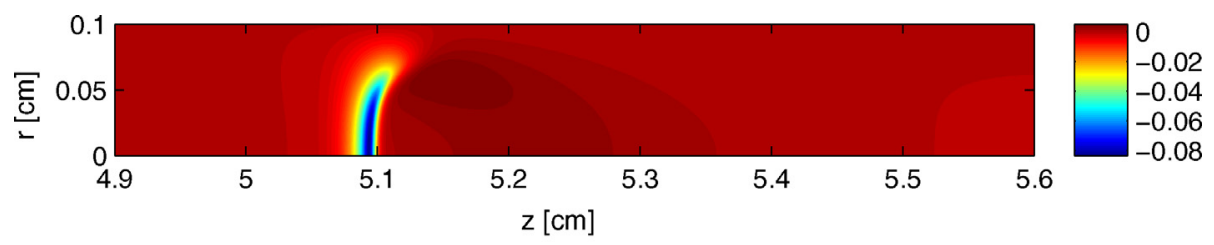

a

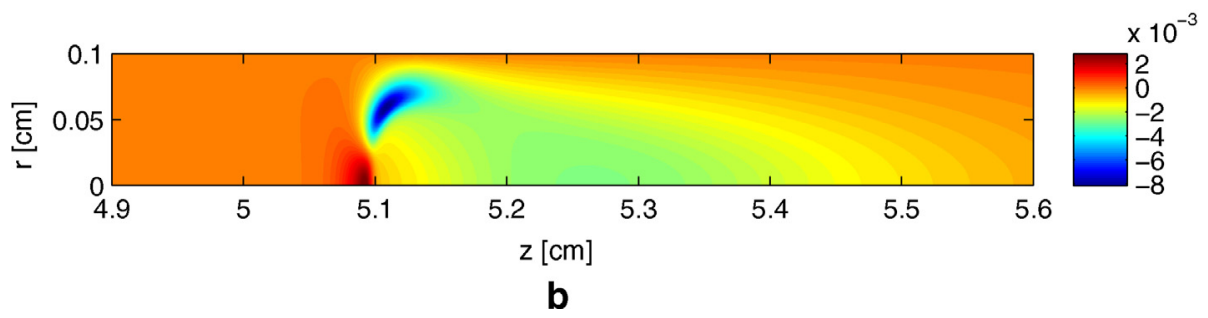

Fig. 19. Temperature fluctuation: (a) real part; and (b) imaginary part.

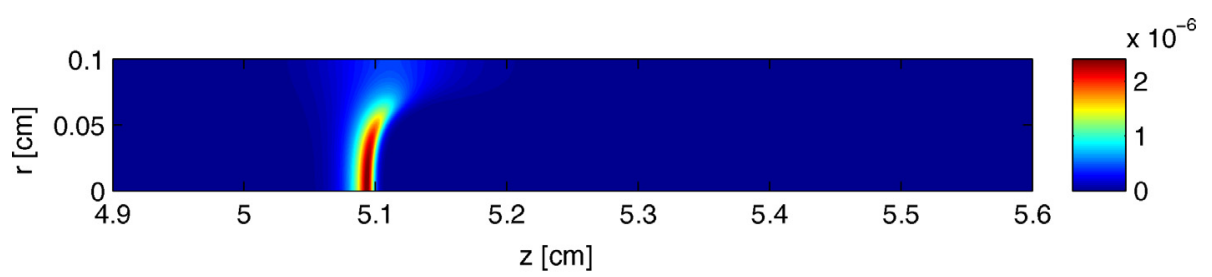

a

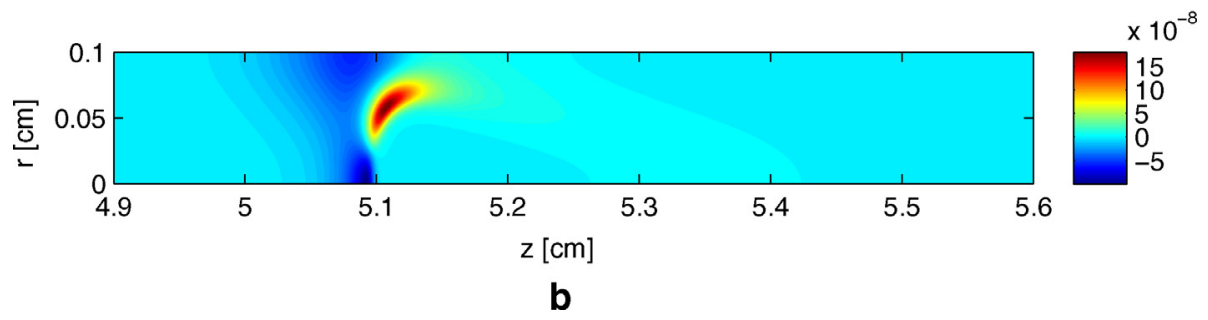

Fig. 20. Concentration fluctuation: (a) real part; and (b) imaginary part. 


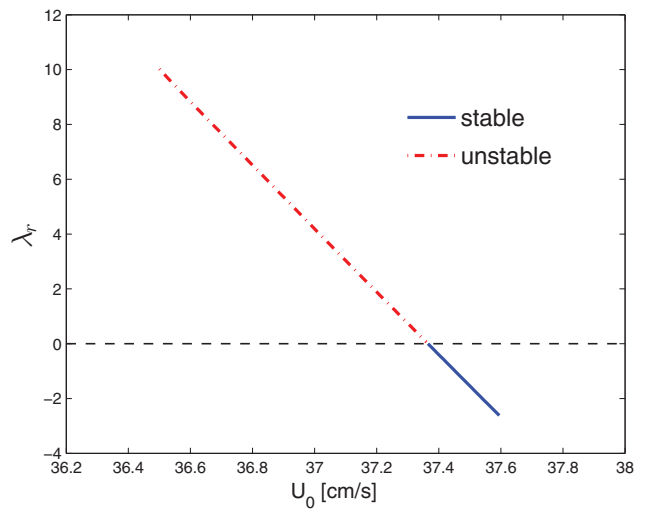

a

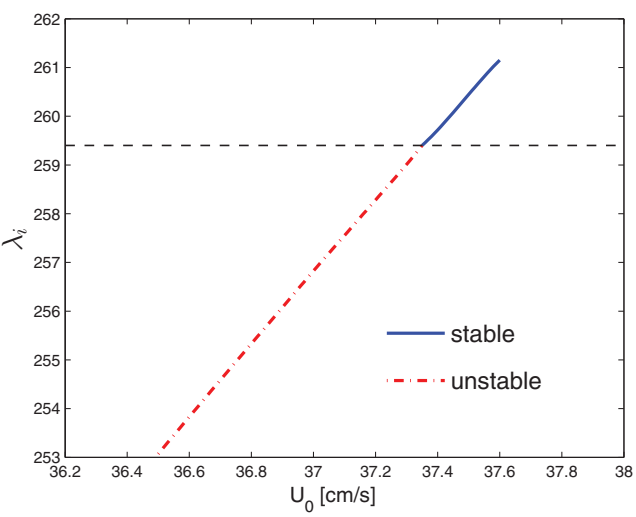

b

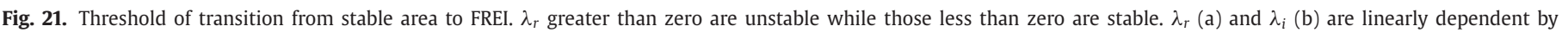
$U_{0}$.

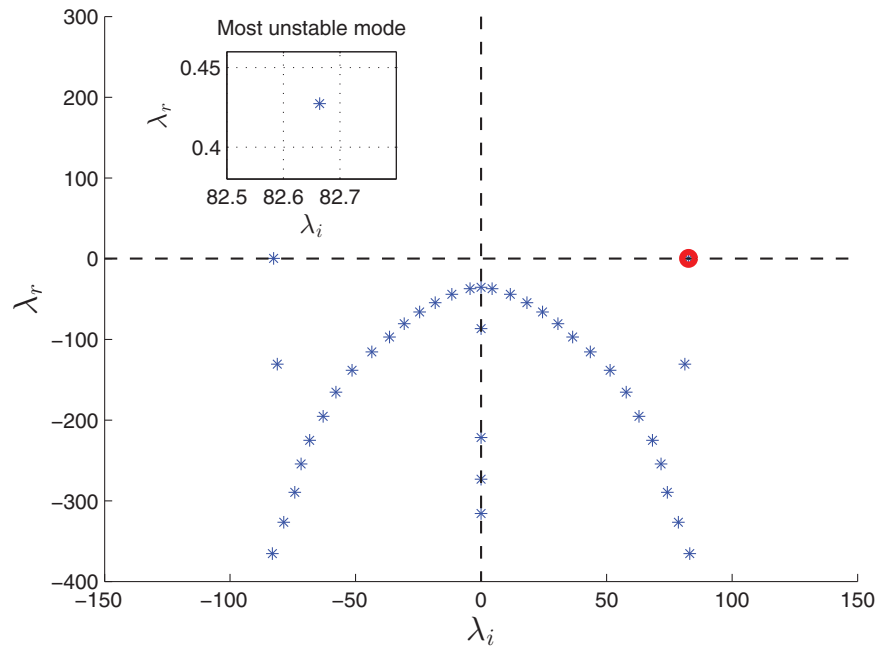

Fig. 22. Spectrum of the 100 most unstable modes. Speed flow is equal to $9.955 \mathrm{~cm} / \mathrm{s}$. A zoom is made on the signed mode.

speed so close to the instability threshold was chosen to be certain that the transition from stable to unstable condition was driven primarily by the linear unstable mode. The temperature and concentration signal obtained at a point close to the ignition is registered. The FREI regime can be observed, with the instability growing exponentially in time, see Fig. 16. The growth rate has been computed to be $\lambda_{r}=0.43$. The Fourier transform of the registered signal does not show a clear principal mode and its harmonics but a richer dynamics, see Fig. 17(a). Therefore, with respect to the
stable/FREI transition at high velocity, the dynamics related to the transition FREI/weak flame is more complex. Yet, for small amplitudes the linear approximation is fulfilled. In fact, considering just the portion of the signal before the appearance of non-linear effects, the Fourier transform displays one mode, Fig. 17(b). Its frequency turns out to be equal to $13 \mathrm{~Hz}$.

In summary, once computed the basic state, the numerical simulation of the linearized set of equations has allowed to compute boththe growth rate and the pulsation for the two instabilities encountered in the phase diagram.

\section{Linear stability analysis}

\subsection{First axisymmetric bifurcation point}

Now we present the results obtained by solving the full eigenvalue problem given by Eq. (12). For $U_{0}=37 \mathrm{~cm} / \mathrm{s}$, the resulting eigenspectrum is shown in Fig. 18. For this speed régime, the basic state turns out to be globally unstable to a small perturbation, furthermore only one mode is found unstable: $\lambda=4.189+i 256.8$. This result is in very good agreement with the numerical results obtained heuristically through numerical simulation, as described in the previous section, $\lambda \simeq 4.2+i 257$. The eigenfunctions associated with this eigenvalue are presented in Figs. 19 and 20 for temperature and concentration fluctuation, respectively.

The temporal oscillation of the physical fluctuation is given by:

$$
\begin{aligned}
\mathbf{q}^{\prime}(r, z, t) & =C_{1} \Re\left[\hat{\mathbf{q}}(r, z) e^{\lambda t}\right] \\
& =C_{1}\left[\hat{\mathbf{q}}_{r}(r, z) \cos (\gamma)-\hat{\mathbf{q}}_{i}(r, z) \sin (\gamma)\right] e^{\lambda_{r} t}
\end{aligned}
$$

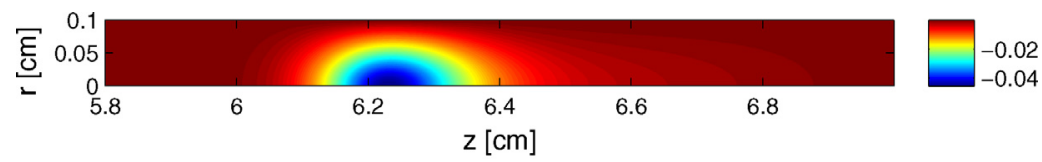

a

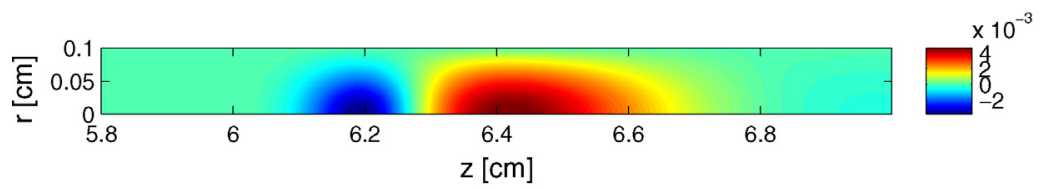

b

Fig. 23. (a) Real part and (b) the imaginary part of the temperature fluctuation. 


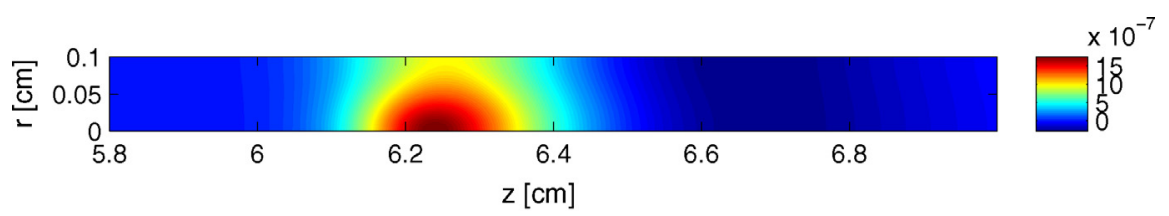

a

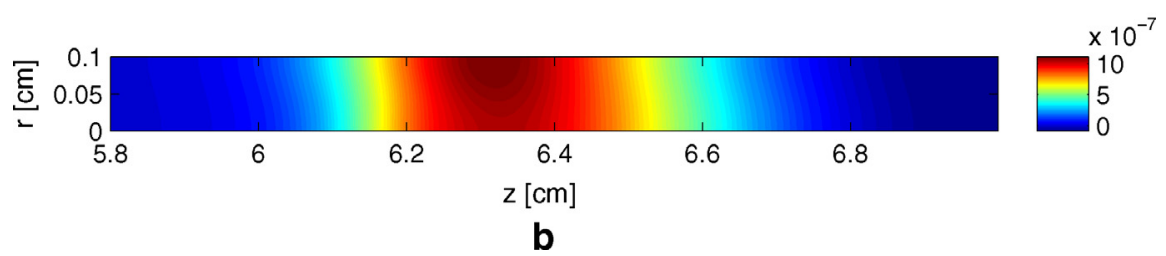

Fig. 24. (a) Real part and (b) the imaginary part of the concentration fluctuation.

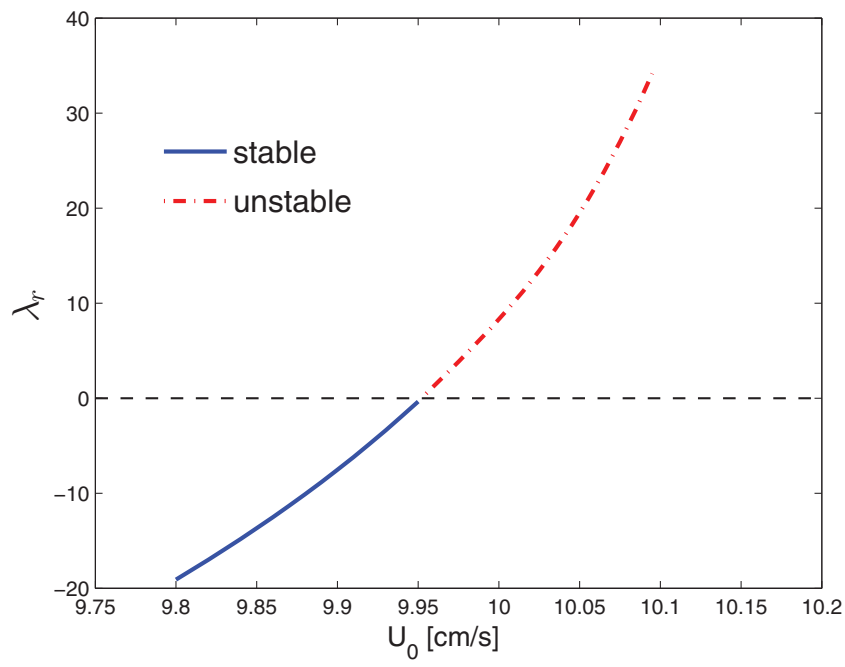

a

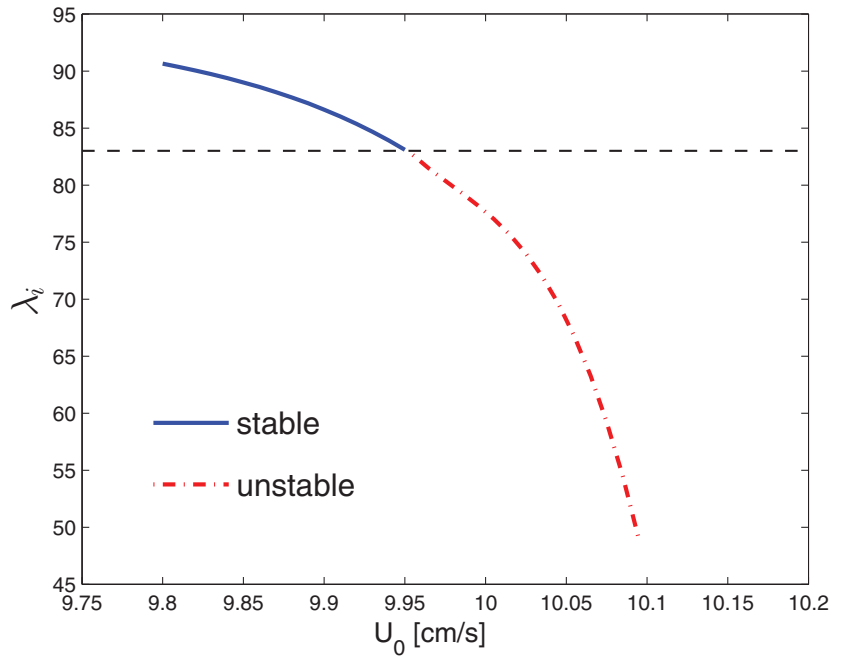

b

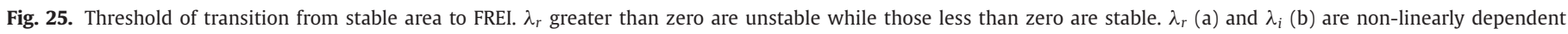
on $U_{0}$.

Table 1

$\sigma$ and $\omega$ comparison obtained by different methods: DNS, LNS and stability analysis.

\begin{tabular}{|c|c|c|c|c|}
\hline & \multicolumn{2}{|c|}{$\begin{array}{l}\text { First bifurcation } \\
U_{0}=37 \mathrm{~cm} / \mathrm{s}\end{array}$} & \multicolumn{2}{|c|}{$\begin{array}{l}\text { Second bifurcation } \\
U_{0}=9.955 \mathrm{~cm} / \mathrm{s}\end{array}$} \\
\hline & $\sigma$ & $\omega$ & $\sigma$ & $\omega$ \\
\hline DNS & 4.2 & 257 & 0.43 & 81.68 \\
\hline LNS & 4.19 & 256.2 & 0.428 & 82.8 \\
\hline SA & 4.189 & 256.8 & 0.427 & 82.64 \\
\hline
\end{tabular}

where $\gamma=\lambda_{i} t$ and $C_{1}=C e^{i \varphi}$ is a complex constant. Using (18) it is possible to reconstruct the time evolution of the fluctuation. It is important to remark that the fluctuations evolve mainly in the radial direction because of the dominating contribution of the radial diffusion.

Then, we have sought for the values of $\lambda_{r}$ and $\lambda_{i}$ as a function of the mixture speed $U_{0}$. Figure 21 shows that the threshold for the transition between the strong stable and the unstable FREI regime, lies between $37.5 / \mathrm{s}\left(\lambda_{r}=0.744\right)$ and $37.4 \mathrm{~cm} / \mathrm{s}\left(\lambda_{r}=-0.3981\right)$. Both $\lambda_{r}$ and $\lambda_{i}$ are found to be linearly dependent on $U_{0}$.

The other modes in Fig. 18 are exponentially dumped as their growth rate is negative $\lambda_{r}<0$. These modes are irrelevant for the asymptotic behavior, but they may contribute to the transient evolution. At variance with the mode shown in Figs. 19 and 20, these modes are not spatially localized, but they are modulated and grow

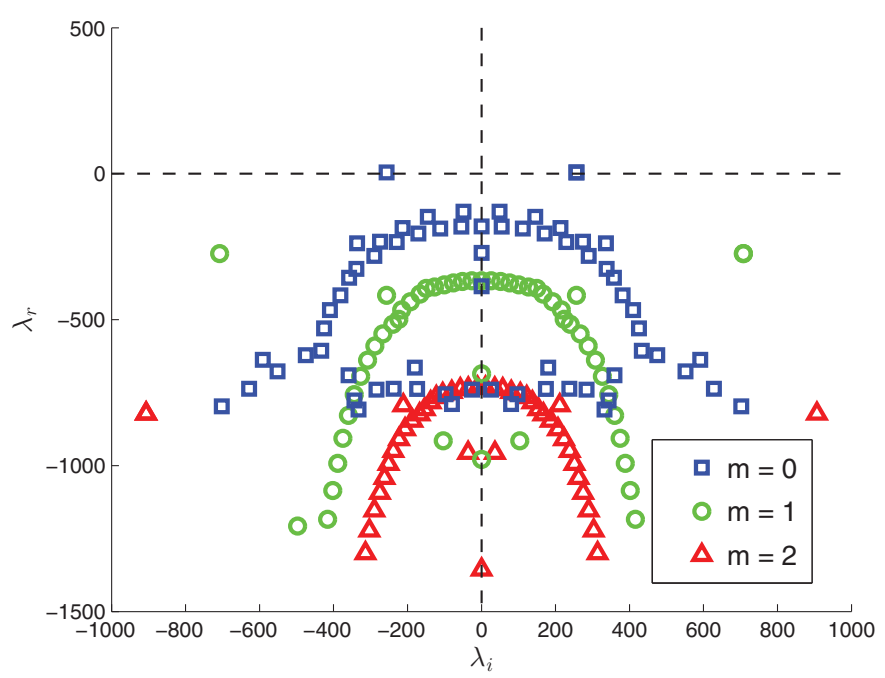

Fig. 26. Eigenspectrum of temporal stability analysis for the azimuthal weave number $m=1(\bigcirc)$ and $m=2(\triangle)$ compared with those found by imposing the wave number $m=0$ ( $\square$ ). Mixture speed $U_{0}=37 \mathrm{~cm} / \mathrm{s}$.

exponentially downstream the flame. Denoting the wavelength $k$, for the stable modes, the phase velocity $v_{p}=\omega / k$ turns out to be $v_{p} \sim U_{0}$. All those modes are therefore generated in the burning region and then simply advected by the flow field. 
Table 2

Unstable mode evolution with $m . U_{0}=$ $37 \mathrm{~cm} / \mathrm{s}$.

\begin{tabular}{lll}
\hline$m$ & $\lambda_{r}$ & $\lambda_{i}$ \\
\hline 0 & 4.189 & 256.8 \\
1 & -273.6 & 707.7 \\
2 & -822.1 & 906.4 \\
\hline
\end{tabular}

\subsection{Second axisymmetric bifurcation point}

Eigenvalue problem (12) has been then solved for the transition between the FREI and the weak flame. Figure 22 shows the eigenspectrum for $U_{0}=9.955 \mathrm{~cm} / \mathrm{s}$. Also in this case only one mode is unstable: $\lambda=0.427+i 82.64$. Again, there is a quite good agreement between the full result and the direct numerical simulation of the linear system, which gave $\lambda \simeq 0.43+i 81.68$. The eigenfunctions associated with the unstable eigenvalue are presented in Figs. 23 and 24, for the temperature and concentration fluctuation, respectively.

The values of $\lambda_{r}$ and $\lambda_{i}$ as a function of the mixture speed $U_{0}$ have been computed. They are shown in Fig. 25. It is possible to see that the threshold lies between $9.95 \mathrm{~cm} / \mathrm{s}\left(\lambda_{r}=-0.361\right)$ and $9.955 \mathrm{~cm} / \mathrm{s}\left(\lambda_{r}=0.427\right)$. Furthermore, both $\lambda_{r}$ and $\lambda_{i}$ turn out to depend exponentially on $U_{0}$, Fig. 25, at variance with the transition stable/FREI, which was linear. Also in this case, a continuous stable branch is present. These modes are just advected by the flow field.

In Table 1 the main results are reported. The growth rates $\sigma$ and the pulsations $\omega$ are compared between the direct numerical simulation (DNS) of the system (1a)-(1b), the numerical simulation of the linearized model (LNS) described in the system (10a)-(10b) and the results of the stability analysis obtained by solving the eigenproblem (12).

\subsection{Non-axisymmetric modes}

Imposing the azimuthal wave number $m$ different to zero it is possible to evaluate the contribution of the $3 \mathrm{D}$ component in the linear stability framework. That seems to be relevant, since nonaxisymmetric modes are found in some numerical works [12,31].

As already said, because of the periodicity of the azimuthal direction, $m$ can assume only integer value $m \in \mathbb{Z}$. The eigenspectrum for the main wave numbers $m=1,2$ is than compared with those

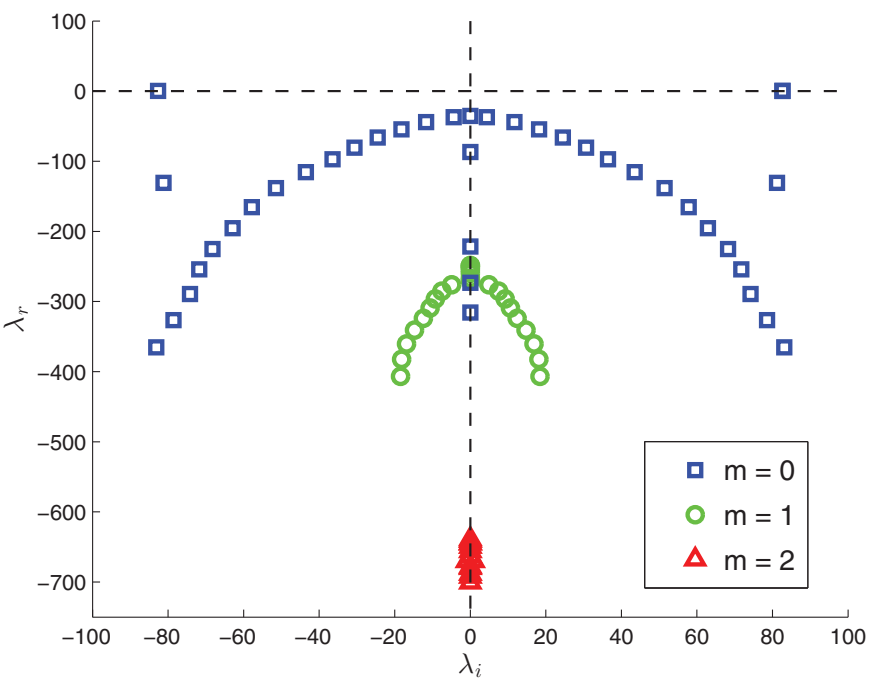

Fig. 28. Eigenspectrum of temporal stability analysis for the azimuthal weave number $m=1(\bigcirc)$ and $m=2(\triangle)$ compared with those found by imposing the wave number $m=0$ ( $\square$ ). Mixture speed $U_{0}=9.955 \mathrm{~cm} / \mathrm{s}$.

found with the wave number $m=0$. For the first instability threshold the results are reported in Fig. 26.

It appears that increasing the value of the azimuthal wave number $m$, the unstable mode becomes stable. The value of the growthrate and the pulsation for the three values of $m$ are reported in Table 2.

The 2D eigenvectors corresponding to the modes in Table 2 are modulated in the $\theta$-direction in order to reconstruct the whole 3D shape of the fluctuation. The result is shown in Fig. 27. Increasing the $m$ value, the perturbation appears to be more localized in the $z$-direction.

For the second stability threshold the eigenspectra for different azimuthal wave number $m$ is shown in Fig. 28. In this case, the stable branch moves toward a more stable region, and the unstable mode disappears. Therefore the model (10) appears to be always stable for $m \neq 0$. At variance with Sánchez-Sanz et al. [25], the first unstable mode turns out to be axisymmetric. This is due to the fact that the pipe shape imposes more constraints, namely the azimuthal periodicity. However, we cannot exclude that changing

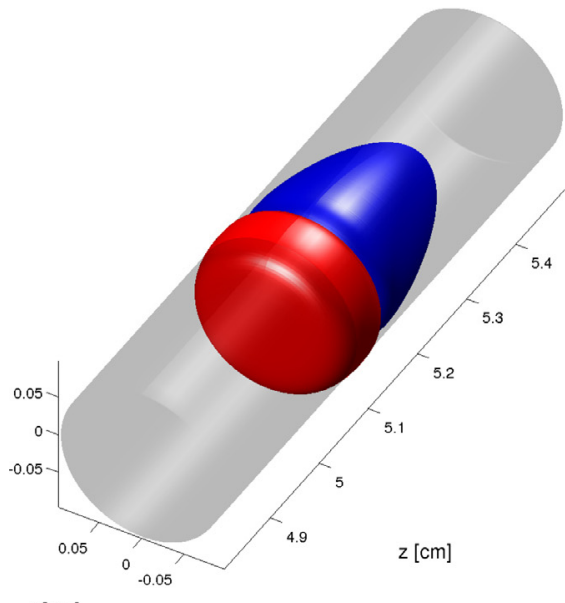

$r[\mathrm{~cm}]$

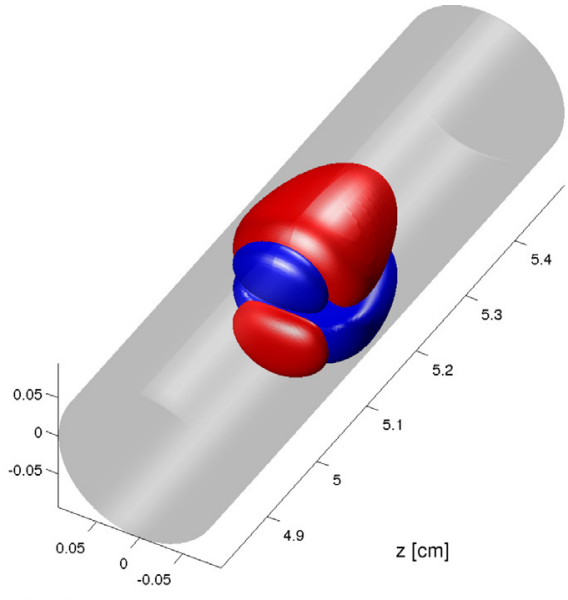

$r[\mathrm{~cm}]$

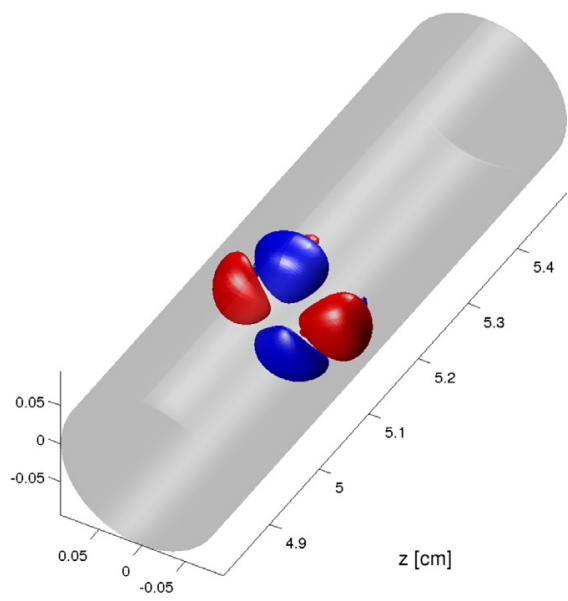

$r[\mathrm{~cm}]$

a

b

Fig. 27. Flame shape for $m=0$ (a), $m=1$ (b) and $m=2$ (c). In the three subfigures the isosurface is $\pm 20 \%$ of the maximum value of the temperature real part. 
the Lewis number or for higher flow-rate the most unstable mode is non-axisymmetric or spinning.

\section{Conclusion}

In this work, we have studied the unstable behavior of a flame arising in the combustion dynamics in a small pipe. In particular, experiments have unfolded the existence of two instability transitions: one from a strong stable flame to a periodic oscillating one (FREI regime); and other from the FREI regime to a weak stable flame at low flow-rate. The problem has been analyzed in the framework of a reduced model. Certainly, with this model the whole dynamic of the flame cannot be represented completely, but it has been recently shown that it is able to reproduce correctly the instabilities in term of flame position, stability threshold and flame shape for both cases. For this reason, we believe that the mechanisms that give rise to the instabilities are properly represented within the proposed model.

Then, once calibrated the present model to reproduce the experimental flame dynamics [18], we have characterized the unstable behavior near the two instability thresholds. For this purpose, we have evaluated the dynamics of the perturbation with respect to the basic state solution, solving the linearized model, which is predictive of the unsteady dynamics near the stability threshold.

Our simulations point out that the main source of the unstable behavior is a phase shift between the production term and the diffusion one. Starting from a stable position and moving in the FREI region, temperature production and radial diffusion show an oscillating behavior in phase opposition. Since integral values provide sufficient information to understand the evolution of the flame, the dynamics is global and does not depend on local behavior. This global feature has been verified through the global stability analysis.

To address this issue, we have computed the whole eigenspectrum with the corresponding eigenvectors. This is our main result. In particular one sole unstable mode has been found. The others are convective modes that enrich the flame dynamics in the transition from the steady solution to the asymptotic one. The growthrate and the circular frequency are in agreement with those computed from direct numerical simulation of the model. Finally, we have also studied the 3D extension of the model. In this way, we have highlighted that only the axisymmetric mode is unstable near the instability threshold. However, the non-axisymmetric behavior is not excluded. The eigenspectra for the azimuthal wave number different from zero show the presence of an isolated global mode that may become unstable in different configurations.

As perspectives, it could be interesting to explain the role of non-linearities when the linear approximation is no longer true. In this way, more sophisticate analysis might be attempted such as linear and non-linear transient growth analysis. These analyses are suggested by the presence of the out-diagonal terms in the Jacobian matrix and also by the presence of convective modes in the eigenspectrum. With the aim of controlling the instability, a wavemaker analysis could also be tried. Lastly, it would be interesting to compare the results of the stability analysis with experiments, even in a different setup.

\section{Acknowledgments}

The authors would like to thank F. Bianco and G. Legros for fruitful discussions.

\section{Appendix A. Linear operator and eigenvalue problem}

In this appendix the definition of the linear operators used in Section 2.5 is given. In order to obtain the fluctuation problem, each physical quantity is divided in the sum of a basic state plus a fluctuation (A.1).

$Y(r, \theta, z, t)=Y_{b}(r, z)+Y^{\prime}(r, \theta, z, t)$

$T(r, \theta, z, t)=T_{b}(r, z)+T^{\prime}(r, \theta, z, t)$

This decomposition is then applied in (1). Since the basic state is stationary, the terms which depend exclusively on the basic state disappear as they are solution of the linearized equations. The fluctuation problem is then described by (A.2a) and (A.2b).

$$
\begin{aligned}
& \frac{\partial Y^{\prime}}{\partial t}+U_{z} \frac{\partial Y^{\prime}}{\partial z}=D\left[\frac{1}{r} \frac{\partial Y^{\prime}}{\partial r}+\frac{\partial^{2} Y^{\prime}}{\partial r^{2}}+\frac{1}{r^{2}} \frac{\partial^{2} Y}{\partial \theta^{2}}+\frac{\partial^{2} Y^{\prime}}{\partial z^{2}}\right]+ \\
& -A e^{\left(-\frac{T_{a}}{T_{b}}\right)} Y^{\prime}-A Y_{b} e^{\left(-\frac{T_{a}}{T_{b}}\right)}\left\{\frac{T_{a}}{T_{b}^{2}}\right\} T^{\prime} \\
& \frac{\partial T^{\prime}}{\partial t}+U_{z} \frac{\partial T^{\prime}}{\partial z}=D\left[\frac{1}{r} \frac{\partial T^{\prime}}{\partial r}+\frac{\partial^{2} T^{\prime}}{\partial r^{2}}+\frac{1}{r^{2}} \frac{\partial^{2} T}{\partial \theta^{2}}+\frac{\partial^{2} T^{\prime}}{\partial z^{2}}\right]+ \\
& +\frac{Q}{C_{p}} A e^{\left(-\frac{T_{a}}{T_{b}}\right)} Y^{\prime}+\frac{Q}{C_{p}} A Y_{b} e^{\left(-\frac{T_{a}}{T_{b}}\right)}\left\{\frac{T_{a}}{T_{b}^{2}}\right\} T^{\prime}
\end{aligned}
$$

In order to simplify the notation, the following operators have been defined:

- Advection operator

$$
\mathcal{A}=U_{z} \frac{\partial}{\partial z}
$$

- Diffusion operator

$$
\mathcal{D}=D\left[\frac{1}{r} \frac{\partial}{\partial r}+\frac{\partial^{2}}{\partial r^{2}}+\frac{1}{r^{2}} \frac{\partial^{2}}{\partial \theta^{2}}+\frac{\partial^{2}}{\partial z^{2}}\right]
$$

- Production operator for $Y$

$$
\mathcal{P}_{Y}\left(T_{b}\right)=A e^{\left(\frac{T_{a}}{T_{b}}\right)}
$$

- Production operator for $T$

$$
\mathcal{P}_{T}\left(Y_{b}, T_{b}\right)=A Y_{b} e^{\left(\frac{T_{a}}{T_{b}}\right)}\left\{\frac{T_{a}}{T_{b}^{2}}\right\}
$$

The symbolic linearized equation for the fluctuation can be written as

$\frac{\partial Y^{\prime}}{\partial t}=\left(-\mathcal{A}+\mathcal{D}-\mathcal{P}_{Y}\left(Y_{b}\right)\right) Y^{\prime}-\mathcal{P}_{T}\left(Y_{b}, T_{b}\right) T^{\prime}$

$\frac{\partial T^{\prime}}{\partial t}=\left(-\mathcal{A}+\mathcal{D}+\frac{Q}{C_{p}} \mathcal{P}_{T}\left(Y_{b}, T_{b}\right)\right) T^{\prime}+\frac{Q}{C_{p}} \mathcal{P}_{Y}\left(Y_{b}\right) Y^{\prime}$

Following Eq. (11) for the global instability, Fourier-Laplace transform is made

$$
\mathbf{q}^{\prime}(r, \theta, z, t)=\hat{\mathbf{q}}(r, z) e^{i m \theta+\lambda t}
$$

Then the obtained equations are:

$\lambda \hat{Y}=\mathcal{L}_{Y}\left(T_{b}, m\right) \hat{Y}-\mathcal{P}_{T}\left(Y_{b}, T_{b}\right) \hat{T}$

$\lambda \hat{T}=\mathcal{L}_{T}\left(Y_{b}, T_{b}, m\right) \hat{T}+\frac{Q}{C_{p}} \mathcal{P}_{Y}\left(T_{b}\right) \hat{Y}$

where $\mathcal{L}_{Y}\left(T_{b}, m\right)=\left(-\mathcal{A}+\mathcal{D}(m)-\mathcal{P}_{Y}\left(T_{b}\right)\right)$ and $\mathcal{L}_{T}\left(Y_{b}, T_{b}, m\right)=$ $\left(-\mathcal{A}+\mathcal{D}(m)+Q \mathcal{P}_{T}\left(Y_{b}, T_{b}\right) / C_{p}\right.$ are the linear operators for the mass 


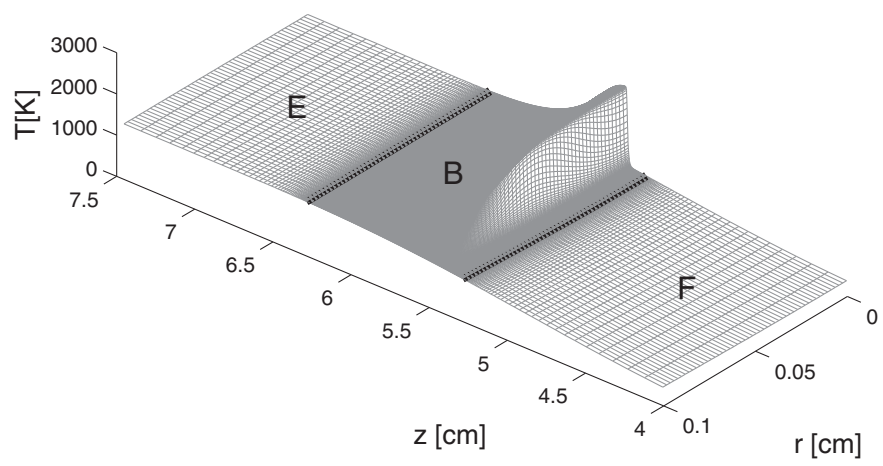

Fig. B.29. Domain discretization on the temperature field in presence of the flame.

fraction and temperature. So the eigenvalue problem can be formulated as (A.9).

$\left(\left[\begin{array}{cc}\mathcal{L}_{Y}\left(T_{b}, m\right) & -\mathcal{P}_{T}\left(Y_{b}, T_{b}\right) \\ \mathcal{Q} \mathcal{P}_{Y}\left(T_{b}\right) / C_{p} & \mathcal{L}_{T}\left(Y_{b}, T_{b}, m\right)\end{array}\right]-\lambda I\right)\left[\begin{array}{l}\hat{Y} \\ \hat{T}\end{array}\right]=0$

In (A.9) the terms off-diagonal, $\mathcal{P}_{T}$ and $-\frac{Q}{C_{p}} \mathcal{P}_{Y}$, could be induce a non-normality in the linear operator. This can be the cause a transient grow of the energy in the system that have to be verified.

\section{Appendix B. Convergence of the eigenvalue problem}

In this appendix, we highlight the influence of the domain size and the mesh setting. A study of the convergence of the mesh has already done by Bianco et al. [14] in his work but we check here the impact of the grid about the well resolution of the unstable eigenvalue. The eigenspectrum of the linear operator in the fluctuation problem strictly depends on the quality of the basic state and the ability of the grid to well discretize the spatial waves. As explained in Section 2.2, to have a reasonably dimension of the linear operator a moving mesh has been adopted. The strategy is the same as that adopted by Bianco et al. [14]. The numerical domain is divided in three parts: burn region (B), fresh air region (F) and exhaust gas (E). The discretization setting is shown in Fig. B.29.

The $\mathrm{B}$ region follows every $n$ steps the position of the flame. The grid size in this zone is constant. Out of the burn region the refinement of the mesh is gradually decreased in space following a geometrical series. In the regions $\mathrm{E}$ and $\mathrm{F}$ the geometrical series is the same. About the spatial resolution of the temperature and concentration field there are three parameters that can influence the result of the stability analysis. The first is the numerical domain dimension, the second is the dimension of the $\mathrm{B}$ region and in the end the minimum grid size in the $B$ region. About the domain dimension, as the asymptotic behavior is piloted by a mode that is localized in the space, even a small dimension around the $B$ region is sufficient to catch the unstable mode. The error on the $\lambda_{r}$ and $\lambda_{i}$ values is less than $10^{-5}$ if a domain between 3 and $8 \mathrm{~cm}$ is used instead of 0 and $10 \mathrm{~cm}$. The domain size is rather linked to the well representation of the all dynamic of the flame from the ignition position to the extinction or stabilized one for the basic state.

The dimension of the $B$ region is the one that is able to well discretize the production zone $\left(\mathcal{P}_{Y}\right.$ and $\left.\mathcal{P}_{T}\right)$. A $\mathrm{B}$ size equal to $0.9 \mathrm{~cm}$ has been used. An error less than $10^{-4}$ is reached if a nonadaptative mesh that has a constant discretization from the ignition point to the stable position of the flame is used.

In the end the axial discretization $d z$ was tested. In the table given below there is the comparison between three grid dimensions for a velocity equal to $37 \mathrm{~cm} / \mathrm{s}$. $d z$ size turns out to be the most critical parameter because it has to be able to catch the production zone that is really tiny as it is possible to see in Fig. 3.

\begin{tabular}{lll}
\hline$d z[\mathrm{~cm}]$ & $\lambda_{r}$ & $\lambda_{i}$ \\
\hline $1.3 \times 10^{-3}$ & 4.1887 & 256.83 \\
$1.6 \times 10^{-3}$ & 4.1878 & 256.79 \\
$2 \times 10^{-3}$ & 4.2661 & 256.53
\end{tabular}

\section{References}

[1] D.C. Walther, J. Ahn, Advances and challenges in the development of power-generation systems at small scales, Prog. Energy Combust. Sci. 37 (2011) 583-610.

[2] N.S. Kaisare, D.G. Vlachos, A review on microcombustion: fundamentals, devices and applications, Prog. Energy Combust. Sci. 38 (2012) 321-359.

[3] K. Maruta, Micro and mesoscale combustion, Proc. Combust. Inst. 33 (2011) 125-150.

[4] A.C. Fernandez-Pello, Micropower generation using combustion: issues and approaches, Proc. Combust. Inst. 29 (2002) 883-899.

[5] D. Dunn-Rankin, E.M. Leal, D.C. Walther, Personal power systems, Prog. Energy Combust. Sci. 31 (2005) 422-465.

[6] S.A. Lloyd, F.J. Weinberg, Three-dimensional effects in counterflow heat-recirculating combustors, Nature 251 (1974) 47-49.

[7] C.-H. Chen, P.D. Ronney, Three-dimensional effects in counterflow heat-recirculating combustors, Proc. Combust. Inst. 33 (2011) 3285-3291.

[8] K. Fu, A.J. Knobloch, F.C. Martinez, D.C. Walther, C. Fernandez-Pello, A.P. Pisano, D. Liepmann, Design and fabrication of a silicon-based MEMS rotary engine, ASME International Mechanical Engineering Congress and Exposition (2001), pp. 875-880.

[9] U. Dogwiler, J. Mantzaras, P. Benz, B. Kaeppeli, R. Bombach, A. Arnold, Homogeneous ignition of methane-air mixtures over platinum: comparison of measurements and detailed numerical predictions, Symp. (Int.) Combust. 27 (1998) 2275-2282.

[10] G. Pizza, C.E. Frouzakis, J. Mantzaras, A.G. Tomboulides, K. Boulouchos, Dynamics of premixed hydrogen/air flames in mesoscale channels, Combust. Flame 155 (2008) 2-20.

[11] C.-H. Tsai, The asymmetric behavior of steady laminar flame propagation in ducts, Combust. Sci. Technol. 180 (2008) 533-545.

[12] G. Pizza, C. Frouzakis, J. Mantzaras, A. Tomboulides, K. Boulouchos, Three-dimensional simulations of premixed hydrogen/air flames in microtubes, J. Fluid Mech. 658 (2010) 463-491.

[13] H. Nakamura, A. Fan, S. Minaev, E. Sereshchenko, R. Fursenko, Y. Tsuboi, K. Maruta, Bifurcations and negative propagation speeds of methane/air premixed flames with repetitive extinction and ignition in a heated microchannel, Combust. Flame 159 (2012) 1631-1643.

[14] F. Bianco, S. Chibbaro, G. Legros, Low-dimensional modeling of flame dynamics in heated microchannels, Chem. Eng. Sci. 122 (2015) 533-544.

[15] K. Maruta, T. Kataoka, N.I. Kim, S. Minaev, R. Fursenko, Characteristics of combustion in a narrow channel with a temperature gradient, Proc. Combust. Inst. 30 (2005) 2429-2436.

[16] F. Richecoeur, D.C. Kyritsis, Experimental study of flame stabilization in low Reynolds and dean number flows in curved mesoscale ducts, Proc. Combust. Inst. 30 (2005) 2419-2427.

[17] T. Jackson, J. Buckmaster, Z. Lu, D. Kyritsis, L. Massa, Flames in narrow circular tubes, Proc. Combust. Inst. 31 (2007) 955-962

[18] Y. Tsuboi, T. Yokomori, K. Maruta, Lower limit of weak flame in a heated channel, Proc. Combust. Inst. 32 (2009) 3075-3081.

[19] V.N. Kurdyumov, J.-M. Truffaut, J. Quinard, A. Wangher, G. Searby, Oscillations of premixed flames in tubes near the flashback conditions, Combust. Sci. Technol. 180 (2008) 731-742

[20] M. Kwon, B. Lee, S. Chung, An observation of near-planar spinning premixed flames in a sudden expansion tube, Combust. Flame 105 (1996) 180-188.

[21] B. Xu, Y. Ju, Experimental study of spinning combustion in a mesoscale divergent channel, Proc. Combust. Inst. 31 (2007) 3285-3292.

[22] V. Kurdyumov, E. Fernndez-Tarrazo, J.-M. Truffaut, J. Quinard, A. Wangher, G. Searby, Experimental and numerical study of premixed flame flashback, Proc. Combust. Inst. 31 (2007) 1275-1282.

[23] V.N. Kurdyumov, G. Pizza, C.E. Frouzakis, J. Mantzaras, Dynamics of premixed flames in a narrow channel with a step-wise wall temperature, Combust. Flame 156 (2009) 2190-2200.

[24] V.N. Kurdyumov, Lewis number effect on the propagation of premixed flames in narrow adiabatic channels: symmetric and non-symmetric flames and their linear stability analysis, Combust. Flame 158 (2011) 1307-1317.

[25] M. Sánchez-Sanz, D. Fernández-Galisteo, V.N. Kurdyumov, Effect of the equivalence ratio, Damköhler number, Lewis number and heat release on the stability of laminar premixed flames in microchannels, Combust. Flame 161 (2014) 1282-1293. 
[26] V. Theofilis, Advances in global linear instability analysis of nonparallel and three-dimensional flows, Prog. Aerosp. Sci. 39 (2003) 249-315.

[27] E. Åkervik, L. Brandt, D.S. Henningson, J. Hœpffner, O. Marxen, P. Schlatter, Steady solutions of the Navier-Stokes equations by selective frequency damping, Phys. Fluids (1994-present) 18 (2006) 068102.

[28] B.E. Jordi, C.J. Cotter, S.J. Sherwin, Encapsulated formulation of the selective frequency damping method, Phys. Fluids (1994-present) 26 (2014) 034101.

[29] L.N. Trefethen, Spectral methods in MATLAB, vol. 10, SIAM, 2000.
[30] E.M. Gennaro, J. Pérez, D. Rodriguez, V. Theofilis, M. Faraco de Medeiros, Development of a code for biglobal linear stability analysis of compressible flows: application to leading-edge boundary layer, 13th Brazilian Congress of Thermal Sciences and Engineering (2010).

[31] G. Pizza, J. Mantzaras, C.E. Frouzakis, A.G. Tomboulides, K. Boulouchos, Suppression of combustion instabilities of premixed hydrogen/air flames in microchannels using heterogeneous reactions, Proc. Combust. Inst. 32 (2009) 3051-3058. 